Pacific Journal of Mathematics

PROOF OF EXTENSIONS OF TWO CONJECTURES ON
STRUCTURAL DAMPING FOR ELASTIC SYSTEMS 


\title{
PROOF OF EXTENSIONS OF TWO CONJECTURES ON STRUCTURAL DAMPING FOR ELASTIC SYSTEMS
}

\author{
Shuping Chen and Roberto Triggiani
}

Let $A$ (the elastic operator) be a positive, self-adjoint operator with domain $D(A)$ in the Hilbert space $X$, and let $B$ (the dissipation operator) be another positive, self-adjoint operator satisfying: $\rho_{1} A^{\alpha} \leq B \leq \rho_{2} A^{\alpha}$ for some constants $0<\rho_{1}<\rho_{2}<\infty$ and $0 \leq \alpha \leq 1$. Consider the operator

$$
\mathscr{A}_{B}=\left|\begin{array}{cc}
0 & I \\
-A & -B
\end{array}\right|
$$

(corresponding to the elastic model $\ddot{x}+B \dot{x}+A x=0$ written as a first order system), which (once closed) is plainly the generator of a strongly continuous semigroup of contractions on the space $E=$ $D\left(A^{1 / 2}\right) \times X$. We prove that if $1 / 2 \leq \alpha \leq 1$, then such semigroup is also analytic (holomorphic) on a triangular sector of $C$ containing the positive real axis. This established a fortiori two conjectures of Goong Chen and David L. Russell on structural damping for elastic systems, which referred to the case $\alpha=1 / 2$. Actually, in the special case $\alpha=1 / 2$ we prove a result stronger than the two conjectures, which yields analyticity of the semigroup over an explicitly identified range of spaces which includes $E$. This latter result was already proved in our previous effort on this problem. Here we provide a technically different and simplified proof of it. We also provide two conceptually and technically different proofs of our main result for $1 / 2 \leq \alpha \leq 1$. Finally, we show that for $0<\alpha<1 / 2$ the semigroup is not analytic.

\section{Introduction, preliminaries, statement of main results.}

\subsection{Introduction. In a recent paper [C-R.1] Goong Chen and} David L. Russell propose a class of mathematical models "exhibiting the empirically observed damping rates in elastic systems." As they show by analyzing the models proposed, the crucial mathematical feature which they seek in order to justify their claim is the property that such models generate strongly continuous, analytic (holomorphic) semigroups. While we refer to [C-R.1] for a discussion of elastic systems and their damping rates as analyzed in past engineering literature, we restrict our interest here to some mathematical questions which are raised in the paper. More specifically, in [C-R.1] Goong Chen and David L. Russell pose two conjectures which-if proven 
correct-would cover precisely the cases that they would like to include in their proposed model for elastic systems. These two conjectures state, qualitatively, that the sought after analyticity property of the underlying dynamics (semigroup) holds true in the case where the dissipation operator is 'comparable' with the $\frac{1}{2}$ th-power of the elastic operator.

In the present paper we study the problem of analyticity of the underlying dynamics (semigroup) raised in [C-R.1], as extended however to the general case where the dissipation operator is 'comparable' with the $\alpha$ th-power of the elastic operator over the entire range $0 \leq \alpha \leq 1$ of the parameter $\alpha$. We prove (Theorem 1.1) that the desired analyticity of the underlying semigroup is indeed attained in the range $\frac{1}{2} \leq \alpha \leq 1$ of the parameter, thereby establishing a fortiori the conjectures of [C-R.1] which refer to the case $\alpha=\frac{1}{2}$. Indeed, in this special case $\alpha=\frac{1}{2}$, we prove a stronger result than the two conjectures (Theorem 1.2), which states that analyticity of the underlying semigroup is in fact obtained over an explicitly identified range of spaces which include the space of interest (the space $E$ in (1.2) below). To complete the analysis, we also prove (§2) that analyticity fails in the range $0<\alpha<\frac{1}{2}$ of the parameter, in line with the well-known case $\alpha=0$, where the semigroup is in fact a group (and therefore cannot be analytic in an infinite dimensional space).

As in [C-R.1], it is assumed throughout that:

(H.1): $A$ (the elastic operator) is a self-adjoint operator on a Hilbert space $X$, strictly positive, with dense domain $D(A)$ and compact resolvent $R(\lambda, A)$, the case of interest in physical applications (one may readily reduce the case of $A$ being only non-negative to the case of $A$ being strictly positive [C-R.1, p. 434]).

(H.2): $B$ (the dissipation operator) is, for the time being, a positive, self-adjoint operator on $X$ likewise with dense domain $D(B)$ in $X$.

Generalizing the mathematical model proposed in [C-R.1] to describe elastic systems, we shall consider in this paper the abstract equation

$$
\ddot{x}+B \dot{x}+A x=0 \text { on } X
$$

or equivalently,

$$
\frac{d}{d t}\left[\begin{array}{c}
x \\
\dot{x}
\end{array}\right]=\mathscr{A}_{B}\left[\begin{array}{c}
x \\
\dot{x}
\end{array}\right] \quad \text { on the space } E=D\left(A^{1 / 2}\right) \times X
$$




$$
\mathscr{A}_{B}=\left[\begin{array}{cc}
0 & I \\
-A & -B
\end{array}\right]
$$

with domain $D\left(\mathscr{A}_{B}\right)$ containing $D(A) \times D(B)$

where the inner product on $E$ is defined by

$$
\left(\left[\begin{array}{l}
x_{1} \\
x_{2}
\end{array}\right],\left[\begin{array}{l}
y_{1} \\
y_{2}
\end{array}\right]\right)_{E}=\left(A^{1 / 2} x_{1}, A^{1 / 2} y_{1}\right)_{X}+\left(x_{2}, y_{2}\right)_{X}
$$

and where the operator $B$ is assumed to satisfy the following additional hypothesis

(H.3): There is a constant $0<\alpha \leq 1$, and there are two constants $0<\rho_{1}<\rho_{2}<\infty$ such that

$$
\text { (1.5a) } \quad \rho_{1} A^{\alpha} \leq B \leq \rho_{2} A^{\alpha} \text {; }
$$

i.e. explicitly

(1.5b) $\rho_{1}\left(A^{\alpha} x, x\right)_{X} \leq(B x, x)_{X} \leq \rho_{2}\left(A^{\alpha} x, x\right)_{X}$,

$$
x \in D\left(B^{1 / 2}\right)=D\left(A^{\alpha / 2}\right) .
$$

Assumption (H.3) is the precise version of our qualitative statement above that $B$ is 'comparable' with $A^{\alpha}$ and is a natural extension of the case $\alpha=\frac{1}{2}$ proposed in [C-R.1]. Thus, the prototype model is the choice $B=2 \rho A^{\alpha}, 0<\rho<\infty, 0<\alpha \leq 1$; i.e. for future reference, the equation

$$
\ddot{x}+2 \rho A^{\alpha} \dot{x}+A x=0 \text { on } X, 0<\rho<\infty ; 0<\alpha \leq 1 ;
$$

or equivalently,

$$
\frac{d}{d t}\left[\begin{array}{c}
x \\
\dot{x}
\end{array}\right]=\mathscr{A}_{\rho \alpha}\left[\begin{array}{c}
x \\
\dot{x}
\end{array}\right] \quad \text { on the space } E=D\left(A^{1 / 2}\right) \times A,
$$

$$
\begin{aligned}
\mathscr{A}_{\rho \alpha} & =\mathscr{A}_{B=2 \rho A^{\prime \prime}}=\left[\begin{array}{cc}
0 & I \\
-A & -2 \rho A^{\alpha}
\end{array}\right], \\
D\left(\mathscr{A}_{\rho \alpha}\right) & =D(A) \times\left[D\left(A^{1 / 2}\right) \cap D\left(A^{\alpha}\right)\right] \\
& = \begin{cases}D(A) \times D\left(A^{1 / 2}\right), & 0<\alpha<\frac{1}{2}, \\
D(A) \times D\left(A^{\alpha}\right), & \frac{1}{2} \leq \alpha \leq 1 .\end{cases}
\end{aligned}
$$

1.2. Preliminaries. We shall collect here some results, to be invoked in subsequent sections, which are either well known or readily verifiable.

(i) In the dissipation-free case $B=0$, the operator

$$
\mathscr{A}_{0}=\left[\begin{array}{cc}
0 & I \\
-A & 0
\end{array}\right], \quad D\left(\mathscr{A}_{0}\right)=D(A) \times D\left(A^{1 / 2}\right)
$$


is skew-adjoint on $E: \mathscr{A}_{0}=-\mathscr{A}_{0}^{*}$ and thus it generates a strongly continuous unitary group $\exp \left(\mathscr{A}_{0} t\right)$ on $E$ (conservative elastic system).

(ii) In the damped case, the operator $\mathscr{A}_{B}$ given by (1.3) is densely defined on $E$ and dissipative here; hence $\mathscr{A}_{B}$ is closeable on $E$, see [P.1, p. 16], [F1, p. 122] and we shall use the same symbol $\mathscr{A}_{B}$ to denote its closure.

(iii) Since $B$ is positive on $X$, then (i) implies that $\mathscr{A}_{B}$ is dissipative on $E$ and the Lumer-Phillips theorem then shows that $\mathscr{A}_{B}$ generates $a$ strongly continuous semigroup of contractions on $E$.

(iv) The resolvent operator $R\left(\lambda, \mathscr{A}_{\rho \alpha}\right)=\left(\lambda I-\mathscr{A}_{\rho \alpha}\right)^{-1}$ of the operator $\mathscr{A}_{\rho \alpha}$ in $(1.8)$ is given by

$$
\begin{gathered}
R\left(\lambda, \mathscr{A}_{\rho \alpha}\right)=\left[\begin{array}{cc}
\frac{I-V_{\rho \alpha}^{-1}(\lambda) A}{\lambda} & V_{\rho \alpha}^{-1}(\lambda) \\
-A V_{\rho \alpha}^{-1}(\lambda) & \lambda V_{\rho \alpha}^{-1}(\lambda)
\end{array}\right], \\
V_{\rho \alpha}(\lambda)=\lambda^{2} I+\lambda 2 \rho A^{\alpha}+A
\end{gathered}
$$

at least for $\operatorname{Re} \lambda>0$. We note that $A$ and $V_{\rho \alpha}(\lambda)$ commute, a property which will be freely used below. Similarly, the resolvent $R\left(\lambda, \mathscr{A}_{B}\right)=$ $\left(\lambda I-\mathscr{A}_{B}\right)^{-1}$ of the operator $\mathscr{A}_{B}$ in (1.3) (and section $\left.1.2(\mathrm{ii})\right)$ is

$$
R\left(\lambda, \mathscr{A}_{B}\right)=\left[\begin{array}{cc}
\frac{I-V_{B}^{-1}(\lambda) A}{\lambda} & V_{B}^{-1}(\lambda) \\
-V_{B}^{-1}(\lambda) A & \lambda V_{B}^{-1}(\lambda)
\end{array}\right],
$$

$$
V_{B}(\lambda)=\lambda^{2} I+\lambda B+A=V_{\rho \alpha}(\lambda)+\lambda\left(B-2 \rho A^{\alpha}\right) \text {, }
$$

$$
\frac{I-V_{B}^{-1}(\lambda) A}{\lambda}=V_{B}^{-1}(\lambda)(\lambda I+B),
$$

at least for $\operatorname{Re} \lambda>0$. We also observe for future use the elementary property that the adjoint $\left[V_{B}^{-1}(\lambda)\right]^{*}$ of $V_{B}^{-1}(\lambda)$ is

$$
\left[V_{B}^{-1}(\lambda)\right]^{*}=V_{B}^{-1}(\bar{\lambda}) \text {. }
$$

REMARK 1.1. Let $\beta \geq 0$. The space $D\left(A^{\beta}\right)$ and its dual $\left[D\left(A^{\beta}\right)\right]^{\prime}$ with respect to the $X$-topology will always be considered as endowed with the following norms

$$
\begin{aligned}
\|x\|_{D\left(A^{\beta}\right)} & =\left\|A^{\beta} x\right\|_{X}, & & x \in D\left(A^{\beta}\right), \\
\|x\|_{\left[D\left(A^{\beta}\right)\right]^{\prime}} & =\left\|A^{-\beta} x\right\|_{X}, & & x \in\left[D\left(A^{\beta}\right)\right]^{\prime}
\end{aligned}
$$

consistently with (1.4). 
REMARK 1.2. With reference to (1.18), we note that the operator $\mathscr{A}_{\rho \alpha}$ is not dissipative on the space $D\left(A^{\alpha}\right) \times X$, which may be suggested by $\mathscr{A}_{\rho \alpha} x=\left[x_{2},-A x_{1}-2 \rho A^{\alpha} x_{2}\right]$ for $\left[x_{1}, x_{2}\right] \in D(A) \times D\left(A^{\alpha}\right)$, unless $\alpha=\frac{1}{2}$. Similarly, the statement 1.2 (iii) on the generation by $\mathscr{A}_{B}$ holds on the fixed space $E$ in (1.2), regardless of the value of $0 \leq \alpha \leq 1$.

The following elementary observation will be invoked in $\S 4$.

LEMMA 1.1. For $0<\rho<\infty ; 0<\alpha \leq 1$; and $A$ as in (H.1), we have for all $x \in X$ and all $\lambda$ with $\operatorname{Re} \lambda>0$ :

$$
\operatorname{Re}\left(\lambda A^{\alpha} V_{\rho \alpha}^{-1}(\lambda) x, x\right)_{X} \geq 0 .
$$

Proof. Immediate from (1.13) after introducing the new variable $\xi(\lambda)=V_{\rho \alpha}^{-1}(\lambda) x$ so that

$$
\operatorname{Re}\left(\lambda A^{\alpha} V_{\rho \alpha}^{-1}(\lambda) x, x\right)=\operatorname{Re}\left(\lambda A^{\alpha} \xi(\lambda),\left(\lambda^{2} I+\lambda 2 \rho A^{\alpha}+A\right) \xi(\lambda)\right) .
$$

In closing, we note that assumption (1.5) is equivalent to the following version

$$
\text { (1.21) } 0<\rho_{1}(y, y)_{X} \leq\left(A^{-\alpha / 2} B A^{-\alpha / 2} y, y\right)_{X} \leq \rho_{2}(y, y)_{X}, \quad y \in X
$$

which suggests the introduction of the operator

$$
S_{\alpha}=A^{-\alpha / 2} B A^{-\alpha / 2}
$$

self-adjoint, bounded, and boundedly invertible on $X$. It is in this form (1.21) that assumption (H.3) will be used below in $\S \S 4,6$.

1.3. Statement of main results. In [C-R.1] G. Chen and D. L. Russell formulated the following two conjectures which refer to the case $\alpha=\frac{1}{2}$.

Assume that the operators $A$ and $B$ satisfy the standing hypotheses (H.1) and (H.2) of $\S 1.1$. Then, the strongly continuous semigroup generated by the operator $\mathscr{A}_{B}$ in (1.3) [see $\S \S 1.2(\mathrm{ii})-(\mathrm{iii})$ ] is also analytic on $E=D\left(A^{1 / 2}\right) \times X$, provided that, in addition:

Conjecture \#1. $\rho_{1}^{2} A \leq B^{2} \leq \rho_{2}^{2} A, 0<\rho_{1}<\rho_{2}<\infty$, i.e., explicitly

$$
\rho_{1}^{2}(A x, x) \leq\left(B^{2} x, x\right) \leq \rho_{2}^{2}(A x, x), \quad x \in D(B)=D\left(A^{1 / 2}\right) ;
$$


or else, provided that, in addition

Conjecture \#2. $\rho_{1} A^{1 / 2} \leq B \leq \rho_{2} A^{1 / 2}, 0<\rho_{1}<\rho_{2}<\infty$; i.e., explicitly

$$
\begin{aligned}
& \rho_{1}\left(A^{1 / 2} x, x\right) \leq(B x, x) \leq \rho_{2}\left(A^{1 / 2} x, x\right) \\
& x \in D\left(B^{1 / 2}\right)=D\left(A^{1 / 4}\right) .
\end{aligned}
$$

An affirmative answer to these two conjectures would imply, in addition to the feature of consistent modelling [C-R.1], also the desirable stability property that all solutions of $(1.1)$, or (1.2), would then decay to zero exponentially as $t \rightarrow+\infty$ in the uniform norm $L(E)$ of $E$. Stated in the language of control theory, this means that the free dynamics (1.1) with $B=0$, which generates a strongly continuous unitary group on $E(\S(1.2)(\mathrm{i}))$, is then 'stabilized' in the uniform norm of $E$ by the feedback action $B \dot{x}$, a most desirable conclusion.

Only partial results in the direction of these conjectures are offered in [C-R.1]. These are:

Corollary 3.2 in [C-R.1] - a local result which states that for each $\rho>0$ there exists $\varepsilon(\rho)>0$ such that if $\rho_{1}=\rho-\varepsilon(\rho)$ and $\rho_{2}=\rho+\varepsilon(\rho)$ in (1.24), then conjecture \#2 holds true; and Theorem 4.1 in [C-R.1] regarding conjecture \#1, which requires however several additional technical assumptions which appear to be difficult to verify.

We note that assumptions (1.23) and (1.24) are not equivalent (unless $A$ and $B$ commute) as mentioned in [C-R.1]; however, it is known that (1.23) implies (1.24), see [K.2, Corollary 7.1 p. 146] and [X.1, p. 5] (Lowner's Theorem). Thus, to give an affirmative response to both conjectures raised in [C-R.1] in the case $\alpha=\frac{1}{2}$, it suffices to study conjecture \#2, the more general of the two.

Indeed, a full description of the problem is provided by the following results.

Theorem 1.1. Assume the standing hypotheses (H.1)-(H.2). Assume, in addition, hypothesis (H.3) with $\frac{1}{2} \leq \alpha \leq 1$ in (1.5).

(a) Then, the strongly continuous semigroup $\exp \left(\mathscr{A}_{B} t\right)$ of contractions generated by the operator $\mathscr{A}_{B}$ in (1.3) [§1.2(ii)-(iii)] is also analytic (holomorphic) on $E=D\left(A^{1 / 2}\right) \times X$.

(b) As a result of part (a), the spectrum determined growth assumption [T.1] is satisfied for $\mathscr{A}_{B}$, and there is a constant $\delta=-\sup \operatorname{Re} \sigma\left(\mathscr{A}_{B}\right)$ $>0, \sigma\left(\mathscr{A}_{B}\right)$ being the spectrum of $\mathscr{A}_{B}$, such that

$$
\left\|e^{\mathscr{A}_{B} t}\right\|_{L(E)} \leq e^{-\delta t}, \quad t \geq 0 .
$$


A negative result on the analyticity of $\exp \left(\mathscr{A}_{B} t\right)$ on $E$ when $0 \leq \alpha<$ $\frac{1}{2}$ is presented in $\S 2$. Spectral properties are given in Appendix $\vec{A}$.

For $\alpha=\frac{1}{2}$ we have a stronger result.

Theorem 1.2. Assume the standing hypotheses (H.1)-(H.2) and, in addition, hypothesis (H.3) for $\alpha=\frac{1}{2}$ (i.e. (1.24)).

(a) Then the operator $\mathscr{A}_{B}$ in (1.3) generates a strongly continuous, analytic semigroup of contractions on each space

$$
E_{\theta}=D\left(A^{3 / 4-\theta / 2}\right) \times D\left(A^{1 / 4-\theta / 2}\right), \quad 0 \leq \theta \leq 1,
$$

topologized as in Remark 1.1, which reduces to the space $E$ in (1.2) for $\theta=\frac{1}{2}$. In (1.26) we have used the conventional notation that for $\beta>0$ the space $D\left(A^{-\beta}\right)$ means $\left[D\left(A^{\beta}\right)\right]^{\prime}$ as in Remark 1.1 .

(b) Moreover, with $\delta$ the same constant as in (1.25), we have

$$
\left\|e^{\mathscr{A}_{B} t}\right\|_{L\left(E_{\theta}\right)} \leq e^{-\delta t}, \quad t \geq 0
$$

Actually, Theorem 1.2 was already established in our first effort on this problem [C-T.1] which referred entirely to the case $\alpha=\frac{1}{2}$. In fact, in this reference we have already provided two different proofs of Theorem 1.2, as well as a precise spectral decomposition of the operator $\mathscr{A}_{\rho \alpha}$ for $\alpha=\frac{1}{2}$ and $0<\rho<1$ as a direct (non-orthogonal) sum of two normal operators on $E$ ([C-T.1, Lemma 2.1]).

REMARK 1.3. It appears that the ideas presented in this paper are susceptible of far reaching generalizations, not fully explored yet. For instance, the arguments of $\S 5$ below (modulo minor variations) permit us to extend the statement of Theorem 1.1 to the following situation: the elastic operator $A$ and the dissipation operator $B$ satisfy hypotheses (H.1)-(H.2) as before, while now $B$ [instead of obeying (1.5)] has domain $\mathscr{D}\left(A^{\alpha_{2}}\right) \subset \mathscr{D}(B) \subset \mathscr{D}\left(A^{\alpha_{1}}\right)$, and satisfies the conditions

$$
\rho_{1}\left(A^{\alpha_{1}} x, x\right) \leq(B x, x), \quad x \in \mathscr{D}(B),
$$

for constants $\frac{1}{2} \leq \alpha_{1} \leq \alpha_{2} \leq 1$, and $0<\rho_{1}, \rho_{2}<\infty$. In fact, $B$ may be relaxed to being essentially self-adjoint provided $B^{*}$ (which is then self-adjoint) satisfies the counterpart of (1.28), (1.29). We intend to present this and related results in a subsequent article. 
REMARK 1.4. Of course, once $\mathscr{A}_{B}$ is an analytic generator, the standard perturbation theory as in [F.1], [P.1], [K.1], [K.2], etc. applies. Thus, the $A$ and $B$ of the present paper may be viewed as principal parts of the actual elastic and dissipation operators.

In the present paper, we shall provide (in $\S 6$ ) a third proof of Theorem 1.2 (the direct proof announced in [C-T.1], below (5.16)). See also Remark 6.1 below. Moreover, we shall provide (in $\S \S 4$ and 5 respectively) two different proofs of Theorem 1.1. It should be noted that our present proofs of Theorem 1.1 in the case $\frac{1}{2} \leq \alpha \leq 1$ are not mere cosmetic variations of our proofs in [C-T.1] of Theorem 1.2 for $\alpha=\frac{1}{2}$ (and in fact, the full strength of Theorem 1.2 is not true for $\frac{1}{2}<\alpha \leq 1$, as for this range of $\alpha$ analyticity attains only on the space $E_{\theta=1 / 2}$ as specified in Theorem 1.1 , not on the entire range of spaces $\left.E_{\theta}, 0 \leq \theta \leq 1\right)$. We have found that the general case $\frac{1}{2}<\alpha \leq 1$ offers new genuine difficulties over the case $\alpha=\frac{1}{2}$. In recent times, the issue of obtaining "structural damping" for the dynamics (1.1) has caught the interest of several authors, e.g. [B.1], [D-P.1], generally in connection with the stabilization of large flexible structures. For instance in reference [B.1, end of $\S 4$ ] A. V. Balakrishnan, apparently unaware of [C-R.1], states: "It would be of interest to develop sufficient conditions on $B$ to assure analyticity," without however advancing any conjecture as [C-R.1] does. Other recent references of interest are [R.2], [R.3].

2. The case: $k_{1} A^{2 \alpha} \leq B^{2} \leq k_{2} A^{2 \alpha}, 0<k_{1}<k_{2}$, with $\alpha<\frac{1}{2}$. The strongly continuous semigroup generated by $\mathscr{A}_{B}$ is not analytic. In this section we shall see that the choice of the power " $A^{1 / 2}$ " as a term of comparison for $B$ is not accidental, in the sense that if the self-adjoint operator $B$ satisfies instead

$$
\begin{aligned}
k_{1}\left\|A^{\alpha} x\right\| \leq\|B x\| \leq k_{2}\left\|A^{\alpha} x\right\|, & 0<k_{1}<k_{2}, \\
x & \in D\left(A^{\alpha}\right)=D(B) \text { for } \alpha<\frac{1}{2}
\end{aligned}
$$

in place of (1.23)-which then implies (1.5) for $\alpha<\frac{1}{2}$ by Lowner's theorem-then the operator $\mathscr{A}_{B}$ in (1.3) [closed as in $\S 1.2(\mathrm{ii})$ ] is the generator of a strongly continuous semi-group on $E$, [see $\S 1.2($ iii)] which however is not analytic in general. Indeed, even more information of negative character is contained in the following construction.

Proposition 2.1. For the positive self-adjoint operator $A$ as in assumption (H.1), denote by $\left\{\mu_{n}\right\}_{n=1}^{\infty}, \mu_{n}>0$, its eigenvalues and by $\left\{e_{n}\right\}_{n=1}^{\infty}$ its corresponding eigenvectors forming an orthonormal basis in 
$X$. Define the operator $B: D(B) \rightarrow X$ by

$$
B e_{n}=b_{n} e_{n}, \quad b_{n}>0
$$

so that $B$ is positive self-adjoint and commutes with $A$. If

$$
\mu_{n} / b_{n}^{2} \rightarrow \infty \text { as } n \uparrow \infty
$$

then the corresponding operator $\mathscr{A}_{B}$ defined by (1.3) (and $\S 1.2(\mathrm{ii})$ ) generates a strongly continuous semigroup on $E$ which, however, is not analytic here.

Proof. Generation by $\mathscr{A}_{B}$ of a strongly continuous semigroup on $E$ was already asserted in $\S 1.2$ (iii). The eigenvalue-vector problem for $\mathscr{A}_{B}$ is

$$
\mathscr{A}_{B}\left|\begin{array}{l}
\Psi_{1} \\
\Psi_{2}
\end{array}\right|=\lambda\left|\begin{array}{l}
\Psi_{1} \\
\Psi_{2}
\end{array}\right| ; \quad \text { i.e. }(-A) \Psi_{1}=\lambda B \Psi_{1}+\lambda^{2} \Psi_{1}
$$

whose solution is given precisely by the eigenvectors $\left\{e_{n}\right\}$ of $A$ :

$$
(-A) e_{n}=\left(\lambda b_{n}+\lambda^{2}\right) e_{n}=-\mu_{n} e_{n} .
$$

Then, the corresponding eigenvalues $\lambda_{n}^{+,-}$of $\mathscr{A}_{B}$ are the solutions of the quadratic equation

$$
\lambda^{2}+b_{n} \lambda+\mu_{n}=0
$$

and are given by

$$
\lambda_{n}^{+,-}=\frac{-b_{n}}{2} \pm i \frac{\sqrt{4 \mu_{n}-b_{n}^{2}}}{2} .
$$

Thus, if (2.3) holds, then $4 \mu_{n}-b_{n}^{2}>0$ for all $n$ sufficiently large and

$$
\left|\frac{\operatorname{Im} \lambda_{n}^{+,-}}{\operatorname{Re} \lambda_{n}^{+,-}}\right|=\sqrt{\frac{4 \mu_{n}}{b_{n}^{2}}-1} \rightarrow \infty
$$

so that the eigenvalues $\left\{\lambda_{n}^{+,-}\right\}$of $\mathscr{A}_{B}$ fail to be contained in a triangular sector of the type

$$
\{\lambda:|\arg (\lambda-a)| \geq \pi / 2+\theta\}
$$

for some real number $a$ and some $\pi / 2>\theta>0$. Thus, as is well 
known [F.1], [P.1], analyticity of the semigroup generated by $\mathscr{A}_{B}$ is out of question.

The case of interest is recaptured as a corollary.

Corollary 2.2. Let $A$ be as in Proposition 2.1 and let $B$ be defined by (2.2), where now

(2.10) $b_{n} \sim \mu_{n}^{\alpha}, \quad \alpha<\frac{1}{2}$, as $n \rightarrow \infty$

$$
\text { (meaning: } c \mu_{n}^{\alpha} \leq b_{n} \leq C \mu_{n}^{\alpha}, 0<c<C \text { ). }
$$

Then, $B$ satisfies (2.1):

(2.11) $\quad c^{2}\left\|A^{\alpha} x\right\|^{2} \leq\|B x\|^{2} \leq C^{2}\left\|A^{\alpha} x\right\|^{2}$,

$$
\alpha<\frac{1}{2}, x \in D\left(A^{\alpha}\right)=D(B)
$$

and $\mathscr{A}_{B}$ generates a strongly continuous semigroup on $E$, which however is not analytic here.

3. Case $\alpha \geq \frac{1}{2}$. The operator $\mathscr{A}_{\rho \alpha}$ is the generator of a strongly continuous, analytic semigroup on $E$. Related properties.

3.1. Statements. By $\S 2$, the strongly continuous semigroup of contractions generated by the operator $\mathscr{A}_{\rho \alpha}$ on $E$ is not analytic here for $0 \leq \alpha<\frac{1}{2}$, as its (point) spectrum is not contained in a triangular sector of the type (2.9). See also Appendix A, in particular Eqs (A.3), (A.4), for the explicit computation of the eigenvalues of $\mathscr{A}_{\rho \alpha}$. Instead, if $\alpha \geq \frac{1}{2}$, the following positive result holds true.

Proposition 3.1. Let $\rho>0$ and $\alpha \geq \frac{1}{2}$ be given.

(i) The strongly continuous semigroup of contractions generated by $\mathscr{A}_{\rho \alpha}$ on $E$ (see $\left.\S 1.2(\mathrm{iii})\right)$ is analytic here.

(ii) With reference to the operator function $V_{\rho \alpha}^{-1}(\lambda)$ defined in (1.13), the following uniform bounds hold true for all $\lambda$ with $\operatorname{Re} \lambda>0$ :

(3.1) $\left\|\lambda^{2} V_{\rho \alpha}^{-1}(\lambda)\right\|_{L(X)}$

$$
\leq C_{\rho \alpha \mu_{1}}= \begin{cases}1 & \text { if } 2 \rho^{2} \mu_{1}^{2 \alpha-1} \geq 1, \\ {\left[4 \rho^{2} \mu_{1}^{2 \alpha-1}\left(1-\rho^{2} \mu_{1}^{2 \alpha-1}\right)\right]^{-1 / 2}} & \text { if } 2 \rho^{2} \mu_{1}^{2 \alpha-1}<1,\end{cases}
$$

$$
\begin{gathered}
\left\|\lambda A^{\alpha} V_{\rho \alpha}^{-1}(\lambda)\right\|_{L(X)} \leq 1 / 2 \rho, \\
\left\|A V_{\rho \alpha}^{-1}(\lambda)\right\|_{L(X)} \leq C_{\rho \alpha \mu_{1}} \quad \text { (same as in (3.1)) }
\end{gathered}
$$

where $(A x, x) \geq \mu_{1}(x, x), \mu_{1}>0$ being the smallest eigenvalue of $A$. 
(iii) By interpolation between (3.2) and (3.3), and between (3.1) and (3.2) respectively, we obtain: for any $0<\theta<1$, the following uniform bounds hold true for all $\lambda$ with $\operatorname{Re} \lambda>0$ :

$$
\begin{gathered}
\left\|\lambda^{1-\theta} A^{(1-\alpha) \theta} A^{\alpha} V_{\rho \alpha}^{-1}(\lambda)\right\|_{L(X)} \leq C_{\rho \alpha \theta}, \\
\left\|\lambda^{2-\theta} A^{\alpha \theta} V_{\rho \alpha}^{-1}(\lambda)\right\|_{L(X)} \leq C_{\rho \alpha \theta} .
\end{gathered}
$$

REMARK 3.1. For future easy reference, we single out the explicit versions of (3.4) and (3.5) for $\theta=\frac{1}{2}$. These will be the only cases which will be invoked in our subsequent analysis in $\S \S 4$ and 6: for all $\lambda$ with $\operatorname{Re} \lambda>0$

$$
\begin{gathered}
\left\|\lambda^{1 / 2} A^{(1+\alpha) / 2} V_{\rho \alpha}^{-1}(\lambda)\right\|_{L(X)} \leq C_{\rho \alpha}, \\
\left\|\lambda^{3 / 2} A^{\alpha / 2} V_{\rho \alpha}^{-1}(\lambda)\right\|_{L(X)} \leq C_{\rho \alpha} .
\end{gathered}
$$

\subsection{Proof of Proposition 3.1.}

Parts (i) and (ii). The following two different proofs may be given for parts (i) and (ii).

The most straightforward strategy consists in first establishing part (ii) by proving directly the uniform bounds $(3.1),(3.2),(3.3)$. Once this is done, by referring back to the explicit expression (1.12) for the resolvent $R\left(\lambda, \mathscr{A}_{\rho \alpha}\right)$, one plainly sees that (3.1), (3.2), and (3.3) collectively state that for all $\lambda$ with $\operatorname{Re} \lambda>0$ and for all $x \in X$ such resolvent satisfies

$$
\left\|R\left(\lambda, \mathscr{A}_{\rho \alpha}\right)_{X}\right\|_{E} \leq \frac{C_{\rho \alpha}}{|\lambda|}\|x\|_{E}, \quad \operatorname{Re} \lambda>0
$$

for some positive constant $C_{\rho \alpha}$. By the sufficiency part of Hille's characterization [F.1, pp. 179-186], [P.1], inequality (3.8) implies that the strongly continuous semigroup of contraction $\exp \left(\mathscr{A}_{\rho \alpha} t\right)($ see $\S 1.2(\mathrm{iii}))$ is also analytic on $E$, thus proving part (i).

A second proof, less direct but also much more informative, inverts the order of the arguments. Here, one first shows part (i) that the semigroup $\exp \left(\mathscr{A}_{\rho \alpha} t\right)$ is analytic on $E$, as a consequence of some interesting spectral properties possessed by the operator $\mathscr{A}_{\rho \alpha}$ plus the location of its spectrum for $\alpha \geq \frac{1}{2}$. (These properties, among other things, yield an explicit spectral expansion for $\exp \left(\mathscr{A}_{\rho \alpha} t\right)$.) Then, by the necessary part of Hille's characterization, it then follows that the 
uniform bound (3.8) for the resolvent holds true. Finally, specializing (3.8) with $x=\left[x_{1}, 0\right]$ and $x=\left[0, x_{2}\right]$ both in $E$ and using the explicit expression (1.12) for $R\left(\lambda, \mathscr{A}_{\rho \alpha}\right)$, one obtains the uniform bounds (3.1), (3.2), (3.3) (without, however, the explicit expression for the constants involved), thus proving part (ii).

The second approach is presented in Appendix A. Here we shall follow the first approach. To establish parts (i) and (ii), it remains to prove (3.1), (3.2), (3.3). Below we shall give a coordinate-free proof of (3.1), (3.2), (3.3), while in Appendix B we give a proof which uses the orthonormal basis of eigenvectors of $A$.

Proof of (3.2). From (1.13)

$$
\frac{V_{\rho \alpha}(\lambda) A^{-\alpha}}{\lambda}=2 \rho I+\lambda A^{-\alpha}+\frac{A^{1-\alpha}}{\lambda}
$$

so that for $x \in D\left(A^{1-\alpha}\right)$

$$
\begin{aligned}
& \left\|\frac{V_{\rho \alpha}(\lambda) A^{-\alpha} x}{\lambda}\right\|^{2}=\|2 \rho x\|^{2}+\left\|\lambda A^{-\alpha} x+\frac{A^{1-\alpha} x}{\lambda}\right\|^{2} \\
& \quad+2 \rho \operatorname{Re}\left(\lambda A^{-\alpha} x+\frac{A^{1-\alpha} x}{\lambda}, x\right) \\
& =4 \rho^{2}\|x\|^{2}+\left\|\lambda A^{-\alpha} x+\frac{A^{1-\alpha} x}{\lambda}\right\|^{2} \\
& \quad+2 \rho(\operatorname{Re} \lambda)\left\|A^{-\alpha / 2} x\right\|^{2}+2 \rho \frac{(\operatorname{Re} \lambda)}{|\lambda|^{2}}\left(A^{1-\alpha} x, x\right) \\
& \geq 4 \rho^{2}\|x\|^{2} \quad \text { for all } \lambda \text { with } \operatorname{Re} \lambda>0 .
\end{aligned}
$$

Moreover, the range of $V_{\rho \alpha}(\lambda) A^{-\alpha} / \lambda$ is all of $X$ for $\operatorname{Re} \lambda>0$ because of (3.10) and since the null space of the adjoint $\left(V_{\rho \alpha}(\lambda) A^{-\alpha} / \lambda\right)^{*}=$ $V_{\rho \alpha}(\bar{\lambda}) A^{-\alpha} / \bar{\lambda}$ (see (1.17)) is plainly the trivial subspace for $\operatorname{Re} \lambda>0$. Thus, this latter property and (3.10) together imply (3.2) as desired. (Note that the present proof does not use $\alpha \geq \frac{1}{2}$.)

Proof of (3.1). From (1.13) with $\alpha \geq \frac{1}{2}$

$$
\text { (3.11) } \frac{V_{\rho \alpha}(\lambda)}{\lambda^{2}}=I+2 \rho \frac{A^{\alpha}}{\lambda}+\frac{A}{\lambda^{2}}=I+2 \rho A^{\alpha-1 / 2}\left(\frac{A^{1 / 2}}{\lambda}\right)+\left(\frac{A^{1 / 2}}{\lambda}\right)^{2}
$$


so that for $x \in D(A)$ we compute

$$
\begin{aligned}
& \left\|\frac{V_{\rho \alpha}(\lambda) x}{\lambda^{2}}\right\|^{2}=\left\|x+2 \rho A^{\alpha-1 / 2} \frac{A^{1 / 2} x}{\lambda}+\left(\frac{A^{1 / 2}}{\lambda}\right)^{2} x\right\|^{2} \\
& =\left\|\left(\frac{A^{1 / 2}}{\lambda}\right)^{2} x\right\|^{2}+4 \rho^{2}\left\|A^{\alpha-1 / 2} \frac{A^{1 / 2} x}{\lambda}\right\|^{2}+\|x\|^{2} \\
& \quad+2 \operatorname{Re}\left(\left(\frac{A^{1 / 2}}{\lambda}\right)^{2} x, x\right)+\mathrm{P}
\end{aligned}
$$

$$
\mathrm{P}=4 \rho\left(\operatorname{Re} \frac{1}{\lambda}\right)\left(\left\|A^{\alpha / 2} x\right\|^{2}+\left\|A^{(\alpha+1) / 2} x\right\|^{2}\right)>0
$$

for $\operatorname{Re} \lambda>0$.

(a) We first let $2 \rho^{2} \mu_{1}^{2 \alpha-1} \geq 1$. It suffices to work only with two terms on the right of (3.12)

$$
\begin{aligned}
2 \operatorname{Re} & \left(\left(\frac{A^{1 / 2}}{\lambda}\right)^{2} x, x\right)+4 \rho^{2}\left\|A^{\alpha-1 / 2} \frac{A^{1 / 2} x}{\lambda}\right\|^{2} \\
& \geq 2\left(\operatorname{Re} \frac{1}{\lambda^{2}}\right)\left\|A^{1 / 2} x\right\|^{2}+\frac{4 \rho^{2} \mu_{1}^{2 \alpha-1}}{|\lambda|^{2}}\left\|A^{1 / 2} x\right\|^{2} \\
= & \left(2 \frac{(\operatorname{Re} \lambda)^{2}-(\operatorname{Im} \lambda)^{2}}{|\lambda|^{4}}+\frac{4 \rho^{2} \mu_{1}^{2 \alpha-1}}{|\lambda|^{2}}\right)\left\|A^{1 / 2} x\right\|^{2} \\
= & \frac{\left\|A^{1 / 2} x\right\|^{2}}{|\lambda|^{4}}\left[\left(4 \rho^{2} \mu_{1}^{2 \alpha-1}+2\right)(\operatorname{Re} \lambda)^{2}\right. \\
& \left.\quad+2\left(2 \rho^{2} \mu_{1}^{2 \alpha-1}-1\right)(\operatorname{Im} \lambda)^{2}\right]>0
\end{aligned}
$$

so that in this case $(3.12)-(3.14)$ yield

$$
\left\|\frac{V_{\rho \alpha}(\lambda) x}{\lambda^{2}}\right\|^{2} \geq\|x\|^{2} \quad \text { for } \operatorname{Re} \lambda>0
$$

and (3.1) is proved in case (a). (The range of $V_{\rho \alpha}(\lambda) / \lambda^{2}$ is all of $X$ for $\operatorname{Re} \lambda>0$ because of (3.15) and since the null space of $\left(V_{\rho \alpha}(\lambda) / \lambda^{2}\right)^{*}=$ $V_{\rho \alpha}(\bar{\lambda}) / \bar{\lambda}^{2}$ is plainly the trivial subspace for $\operatorname{Re} \lambda>0$.) 
(b) We now let $2 \rho^{2} \mu_{1}^{2 \alpha-1}<1$. We now work with the first four terms on the right of (3.12), which we re-write as follows

$$
\begin{aligned}
\text { (3.16) } \| & \frac{V_{\rho \alpha}(\lambda) x}{\lambda^{2}} \|^{2} \\
\geq & \left(\left(\frac{A^{1 / 2}}{|\lambda|}\right)^{4} x, x\right)+4 \rho^{2} \mu_{1}^{2 \alpha-1}\left(\left(\frac{A^{1 / 2}}{|\lambda|}\right)^{2} x, x\right) \\
& +(x, x)-2\left(\left(\frac{A^{1 / 2}}{|\lambda|}\right)^{2} x, x\right) \\
(3.17)= & \left(\left[\left(\frac{A^{1 / 2}}{|\lambda|}\right)^{2}-I\right]^{2} x, x\right)+4 \rho^{2} \mu_{1}^{2 \alpha-1}\left(\left(\frac{A^{1 / 2}}{|\lambda|}\right)^{2} x, x\right) \\
(3.18)= & \left(\left[\left(\frac{A^{1 / 2}}{|\lambda|}\right)^{2}-I\right]^{2} x, x\right) \\
& +4 \rho^{2} \mu_{1}^{2 \alpha-1}\left(\left[\left(\frac{A^{1 / 2}}{|\lambda|}\right)^{2}-I\right] x, x\right)^{2}+4 \rho^{2} \mu_{1}^{2 \alpha-1}\|x\|^{2} \\
= & \left(\left\{\left[\left(\frac{A^{1 / 2}}{|\lambda|}\right)^{2}-I\right]+2 \rho^{2} \mu_{1}^{2 \alpha-1} I\right\}^{2} x, x\right) \\
& +4 \rho^{2} \mu_{1}^{2 \alpha-1}\|x\|^{2}-\left(2 \rho^{2} \mu_{1}^{2 \alpha-1}\right)^{2}\|x\|^{2}
\end{aligned}
$$

(dropping the first (positive) term)

$$
\geq 4 \rho^{2} \mu_{1}^{2 \alpha-1}\left(1-\rho^{2} \mu_{1}^{2 \alpha-1}\right)\|x\|^{2}
$$

uniformly in $\lambda$ for $\operatorname{Re} \lambda>0$. Thus (3.20) proves inequality (3.1), as desired, also in case (b).

Proof of (3.3). From (3.13) with $\alpha \geq \frac{1}{2}$

$$
\begin{aligned}
V_{\rho \alpha}(\lambda) A^{-1} & =I+2 \rho \lambda A^{\alpha-1}+\lambda^{2} A^{-1} \\
& =I+2 \rho A^{\alpha-1 / 2}\left(\lambda A^{-1 / 2}\right)+\left(\lambda A^{-1 / 2}\right)^{2} .
\end{aligned}
$$

The proof now proceeds as for (3.1) from (3.11) through (3.20), with the expression $\left(A^{1 / 2} /|\lambda|\right)$ in that proof replaced by $\left(\lambda A^{-1 / 2}\right)$ now. We only sketch it. Let $x \in X$. Then

$$
\begin{aligned}
\left\|V_{\rho \alpha}(\lambda) A^{-1} x\right\|^{2}= & \left\|\left(\lambda A^{-1 / 2}\right)^{2} x\right\|^{2}+4 \rho^{2}\left\|A^{\alpha-1 / 2}\left(\lambda A^{-1 / 2}\right) x\right\|^{2} \\
& +\|x\|^{2}+2 \operatorname{Re}\left(\left(\lambda A^{-1 / 2}\right)^{2} x, x\right)+\mathbb{P}
\end{aligned}
$$


counterpart of (3.12), where now

$$
\mathrm{P}=4 \rho(\operatorname{Re} \lambda)\left(\left\|A^{(\alpha-1) / 2} x\right\|^{2}+\left\|\lambda A^{(\alpha-2) / 2} x\right\|^{2}\right)>0
$$

for $\operatorname{Re} \lambda>0$.

(a) We first let $2 \rho^{2} \mu_{1}^{2 \alpha-1} \geq 1$. Here, again, we work with only two terms

$$
\begin{aligned}
2 \operatorname{Re}( & \left.\left(\lambda A^{-1 / 2}\right)^{2} x, x\right)+4 \rho^{2}\left\|A^{\alpha-1 / 2}\left(\lambda A^{-1 / 2}\right) x\right\|^{2} \\
\geq & 2\left(\operatorname{Re} \lambda^{2}\right)\left\|A^{-1 / 2} x\right\|^{2}+4 \rho^{2} \mu_{1}^{2 \alpha-1}|\lambda|^{2}\left\|A^{-1 / 2} x\right\|^{2} \\
= & {\left[\left(4 \rho^{2} \mu_{1}^{2 \alpha-1}+2\right)(\operatorname{Re} \lambda)^{2}\right.} \\
& \left.\quad+2\left(2 \rho^{2} \mu_{1}^{2 \alpha-1}-1\right)(\operatorname{Im} \lambda)^{2}\right]\left\|A^{-1 / 2} x\right\|^{2}>0
\end{aligned}
$$

counterpart of (3.14).

(b) We now let $2 \rho^{2} \mu_{1}^{2 \alpha-1}<1$ and work with the first four terms on the right of (3.22). We re-write them as follows

$$
\begin{aligned}
& \left\|V_{\rho \alpha}(\lambda) A^{-1} x\right\|^{2} \\
& \geq\left(\left(|\lambda| A^{-1 / 2}\right)^{4} x, x\right)+4 \rho^{2} \mu_{1}^{2 \alpha-1}\left(\left(|\lambda| A^{-1 / 2}\right)^{2} x, x\right) \\
& \quad+(x, x)-2\left(\left(|\lambda| A^{-1 / 2}\right)^{2} x, x\right) \quad(\text { counterpart of }(3.16))
\end{aligned}
$$

$$
\begin{aligned}
= & \left(\left[\left(|\lambda| A^{-1 / 2}\right)^{2}-I\right]^{2} x, x\right) \\
& +4 \rho^{2} \mu_{1}^{2 \alpha-1}\left(\left[\left(|\lambda| A^{-1 / 2}\right)^{2}-I\right] x, x\right)+4 \rho^{2} \mu_{1}^{2 \alpha-1}\|x\|^{2}
\end{aligned}
$$$$
=\left(\left\{\left[\left(|\lambda| A^{-1 / 2}\right)^{2}-I\right]+2 \rho^{2} \mu_{1}^{2 \alpha-1} I\right\}^{2} x, x\right)
$$$$
+4 \rho^{2} \mu_{1}^{2 \alpha-1}\|x\|^{2}-\left(2 \rho^{2} \mu_{1}^{2 \alpha-1}\right)^{2}\|x\|^{2}
$$

(counterpart of (3.19))

(3.27)

$$
\geq 4 \rho^{2} \mu_{1}^{2 \alpha-1}\left(1-\rho^{2} \mu_{1}^{2 \alpha-1}\right)\|x\|^{2}
$$

uniformly in $\lambda$ with $\operatorname{Re} \lambda>0$ and the proof is complete.

Part (iii). This follows by standard interpolation argument, using the moment inequality, e.g. [K.2]. Details are omitted.

4. Case $\frac{1}{2} \leq \alpha \leq 1$. A first proof of Theorem 1.1. Our goal is to show that there exists a positive constant $K$ such that the following 
uniform bound holds true for all $\lambda$ with $\operatorname{Re} \lambda>0$ :

$$
\begin{aligned}
& \left\|\lambda R\left(\lambda, \mathscr{A}_{B}\right)\right\|_{L(E)} \\
& \quad=\left\|\left[\begin{array}{cc}
A^{1 / 2} & 0 \\
0 & I
\end{array}\right]\left[\begin{array}{cc}
I-V_{B}^{-1}(\lambda) A & \lambda V_{B}^{-1}(\lambda) \\
-\lambda V_{B}^{-1}(\lambda) A & \lambda^{2} V_{B}^{-1}(\lambda)
\end{array}\right]\left[\begin{array}{cc}
A^{-1 / 2} & 0 \\
0 & I
\end{array}\right]\right\|_{L(W)} \\
& \quad \leq K .
\end{aligned}
$$

$W=X \times X$, see (1.18). Once (4.1) is established, standard semi-group theory [F.1] [P.1] guarantees that, as desired, the strongly continuous semigroup of contractions generated by $\mathscr{A}_{B}$ on $E$ (see $(1.2(\mathrm{iii}))$ is, in fact, analytic here. Inequality (4.1) is in turn equivalent to the following set of three inequalities: there exists a positive constant $M$ such that the following uniform bounds hold true for all $\lambda$ with $\operatorname{Re} \lambda>0$ :

$$
\begin{gathered}
\left\|A^{1 / 2} V_{B}^{-1}(\lambda) A^{1 / 2}\right\|_{L(X)} \leq M, \\
\left\|\lambda A^{1 / 2} V_{B}^{-1}(\lambda)\right\|_{L(X)} \leq M, \\
\left\|\lambda^{2} V_{B}^{-1}(\lambda)\right\|_{L(X)} \leq M .
\end{gathered}
$$

In fact, let $a_{i j}, i, j=1,2$, denote the entries of the matrix $\lambda R\left(\lambda, \mathscr{A}_{B}\right)$. Then, (4.2), (4.3), and (4.4) refer directly to $a_{11}, a_{12}$, and $a_{22}$, while $a_{21}$ yields $\lambda V_{B}^{-1}(\lambda) A^{1 / 2}$ whose uniform bound in $L(X)$ for $\operatorname{Re} \lambda>0$ is equivalent to that of the adjoint operator $\left(\lambda V_{B}^{-1}(\lambda) A^{1 / 2}\right)^{*}=$ $\bar{\lambda} A^{1 / 2} V_{B}^{-1}(\bar{\lambda})$, see $(1.17)$, for $\operatorname{Re} \bar{\lambda}=\operatorname{Re} \lambda>0$, in turn equivalent to (4.3).

4.1. Proof of (4.2). A Fundamental Lemma. Since

$$
V_{\rho \alpha}^{-1}(\lambda)-V_{B}^{-1}(\lambda)=V_{\rho \alpha}^{-1}(\lambda)\left(V_{B}(\lambda)-V_{\rho \alpha}(\lambda)\right) V_{B}^{-1}(\lambda)
$$

we compute

$$
\begin{aligned}
& A^{1 / 2} V_{\rho \alpha}^{-1}(\lambda) A^{1 / 2}-A^{1 / 2} V_{B}^{-1}(\lambda) A^{1 / 2} \\
& \quad=A^{1 / 2} V_{\rho \alpha}^{-1}(\lambda)\left(B-2 \rho A^{\alpha}\right) \lambda V_{B}^{-1}(\lambda) A^{1 / 2} \\
& \quad=A^{1 / 2+\alpha / 2} V_{\rho \alpha}^{-1}(\lambda)\left(A^{-\alpha / 2} B A^{-\alpha / 2}-2 \rho I\right) \lambda A^{\alpha / 2} V_{B}^{-1}(\lambda) A^{1 / 2} .
\end{aligned}
$$

If we set, as in (1.22),

$$
S_{\alpha}=A^{-\alpha / 2} B A^{-\alpha / 2} \in L(X),
$$

then $S_{\alpha}$ is a bounded self-adjoint operator on $X$, boundedly invertible here, by assumption (1.5). Distributing $\lambda$ across in (4.6), we re-write (4.6) as

$$
\text { (4.8) } \begin{aligned}
A^{1 / 2} V_{\rho \alpha}^{-1}(\lambda) A^{1 / 2}-A^{1 / 2} V_{B}^{-1}(\lambda) A^{1 / 2} \\
=\lambda^{1 / 2} A^{1 / 2+\alpha / 2} V_{\rho \alpha}^{-1}(\lambda)\left(S_{\alpha}-2 \rho I\right) \lambda^{1 / 2} A^{\alpha / 2} V_{B}^{-1}(\lambda) A^{1 / 2}
\end{aligned}
$$


But, by (3.3) and (3.6), the two terms $A^{1 / 2} V_{\rho \alpha}^{-1}(\lambda) A^{1 / 2}$ and $\lambda^{1 / 2} A^{1 / 2+\alpha / 2} V_{\rho \alpha}^{-1}(\lambda)$ are uniformly bounded in $L(X)$ for all $\lambda$ with $\operatorname{Re} \lambda>0$. Thus, identity (4.8) reveals that the desired uniform bound (4.2) for $A^{1 / 2} V_{B}^{-1}(\lambda) A^{1 / 2}$ holds true as soon as we establish that $\lambda^{1 / 2} A^{\alpha / 2} V_{B}^{-1}(\lambda) A^{1 / 2}$ is uniformly bounded in $L(X)$ for all $\lambda$ with $\operatorname{Re} \lambda>$ 0 . This is then the special case $\beta=1$ of the following Proposition, which we state in greater generality at no extra effort.

Proposition 4.1. With $\frac{1}{2} \leq \alpha \leq 1$ given, let $\alpha<\beta \leq 1$. Then, there exists a positive constant $k_{\alpha \beta}$ such that the following uniform bound holds true for all $\lambda$ with $\operatorname{Re} \lambda>0$ :

$$
\left\|\lambda^{1+(\alpha-\beta) / 2(1-\alpha)} A^{\alpha / 2} V_{B}^{-1}(\lambda) A^{\beta / 2}\right\| \leq k_{\alpha \beta} .
$$

Proof of Proposition 4.1. By (4.5) and (4.7)

$$
\begin{aligned}
\lambda^{1+} & (\alpha-\beta) / 2(1-\alpha) A^{\alpha / 2} V_{\rho \alpha}^{-1}(\lambda) A^{\beta / 2} \\
& -\lambda^{1+(\alpha-\beta) / 2(1-\alpha)} A^{\alpha / 2} V_{B}^{-1}(\lambda) A^{\beta / 2} \\
= & \lambda^{1+(\alpha-\beta) / 2(1-\alpha)} A^{\alpha / 2}\left(B-2 \rho A^{\alpha}\right) \lambda V_{B}^{-1}(\lambda) A^{\beta / 2} \\
= & \lambda A^{\alpha} V_{\rho \alpha}^{-1}(\lambda)\left(S_{\alpha}-2 \rho I\right) \lambda^{1+(\alpha-\beta) / 2(1-\alpha)} A^{\alpha / 2} V_{B}^{-1}(\lambda) A^{\beta / 2}
\end{aligned}
$$

from which

$$
\begin{aligned}
& \lambda^{1+(\alpha-\beta) / 2(1-\alpha)} A^{\alpha / 2} V_{\rho \alpha}^{-1}(\lambda) A^{\beta / 2} \\
& =\left[I+\lambda A^{\alpha} V_{\rho \alpha}^{-1}(\lambda)\left(S_{\alpha}-2 \rho I\right)\right] \lambda^{1+(\alpha-\beta) / 2(1-\alpha)} A^{\alpha / 2} V_{B}^{-1}(\lambda) A^{\beta / 2} .
\end{aligned}
$$

The following Lemma is fundamental.

LEMMA 4.2. Let $0<2 \rho<\rho_{1}$, see (1.5). Then the operator

$$
\mathscr{G}_{\rho \alpha}(\lambda)=I+\lambda A^{\alpha} V_{\rho \alpha}^{-1}(\lambda)\left(S_{\alpha}-2 \rho I\right)
$$

is bounded and boundedly invertible on $L(X)$ (isomorphism), uniformly in $\operatorname{Re} \lambda>0$; in particular, there is a positive constant $c_{\rho \alpha}$ such that for all $\lambda$ with $\operatorname{Re} \lambda>0$ we have

$$
\begin{aligned}
\left\|\mathscr{G}_{\rho \alpha}^{-1}(\lambda)\right\|_{L(X)} & =\left\|\left[I+\lambda A^{\alpha} V_{\rho \alpha}^{-1}(\lambda)\left(S_{\alpha}-2 \rho I\right)\right]^{-1}\right\|_{L(X)} \\
& \leq c_{\rho \alpha} .
\end{aligned}
$$

Assuming for the time being the validity of (4.13), we then obtain from

$$
\begin{aligned}
& \lambda^{1+(\alpha-\beta) / 2(1-\alpha)} A^{\alpha / 2} V_{B}^{-1}(\lambda) A^{\beta / 2} \\
& \quad=\mathscr{G}_{\rho \alpha}^{-1}(\lambda) \lambda^{1+(\alpha-\beta) / 2(1-\alpha)} A^{\alpha / 2} V_{\rho \alpha}^{-1}(\lambda) A^{\beta / 2}
\end{aligned}
$$


and we need to show that the left hand side of (4.14) is uniformly bounded in $L(X)$ for $\operatorname{Re} \lambda>0$. To establish this, since $(\alpha+\beta) / 2>\alpha$ under the present assumption, we appeal to the uniform bound (3.4) with $\theta$ identified by $(1-\alpha) \theta+\alpha=(\alpha+\beta) / 2$. Thus

$$
\theta=\frac{\alpha-\beta}{2(1-\alpha)} \text { and } 1-\theta=1+\frac{\alpha-\beta}{2(1-\alpha)}
$$

and our claim follows. Then (3.4) and (4.13) applied to (4.14) prove (4.9) as desired.

Proof of Lemma 4.2. Let $0<2 \rho<\rho_{1}$. From assumption (1.5), we see that the operator $S_{\alpha}$ in (4.7) satisfies

$$
0<\left(\rho_{1}-2 \rho\right) I \leq S_{\alpha}-2 \rho I \leq\left(\rho_{2}-2 \rho\right) I
$$

and hence also

$$
\frac{1}{\rho_{2}-2 \rho} I \leq\left(S_{\alpha}-2 \rho I\right)^{-1} \leq \frac{1}{\rho_{1}-2 \rho} I
$$

since $S_{\alpha}-2 \rho I \in L(X)$ is self-adjoint. Then, if we rewrite (4.12) as

$$
\mathscr{G}_{\rho \alpha}(\lambda)=\left(\left(S_{\alpha}-2 \rho I\right)^{-1}+\lambda A^{\alpha} V_{\rho \alpha}^{-1}(\lambda)\right)\left(S_{\alpha}-2 \rho I\right)
$$

we see by (4.17) that if we can show the uniform bound

$$
\left\|\left(\left(S_{\alpha}-2 \rho I\right)^{-1}+\lambda A^{\alpha} V_{\rho \alpha}^{-1}(\lambda)\right)^{-1}\right\|_{L(X)} \leq \rho_{2}-2 \rho
$$

for all $\lambda$ with $\operatorname{Re} \lambda>0$, then (4.13) is established as desired. To this end we compute for $x \in X$

$$
\begin{aligned}
&\left\|\left(\left(S_{\alpha}-2 \rho I\right)^{-1}+\lambda A^{\alpha} V_{\rho \alpha}^{-1}(\lambda)\right) x\right\|\|x\| \\
& \geq\left|\left(\left(S_{\alpha}-2 \rho I\right)^{-1} x, x\right)+\left(\lambda A^{\alpha} V_{\rho \alpha}^{-1}(\lambda) x, x\right)\right| \\
&=\left\{\left[\left(\left(S_{\alpha}-2 \rho I\right)^{-1} x, x\right)+\right.\right.\left.\operatorname{Re}\left(\lambda A^{\alpha} V_{\rho \alpha}^{-1}(\lambda) x, x\right)\right]^{2} \\
&+ {\left.\left[\operatorname{Im}\left(\lambda A^{\alpha} V_{\rho \alpha}^{-1}(\lambda) x, x\right)\right]^{2}\right\}^{1 / 2} }
\end{aligned}
$$

(using (1.20) of Lemma 1.1 and (4.17))

$$
\geq\left(\left(S_{\alpha}-2 \rho I\right)^{-1} x, x\right) \geq \frac{1}{\rho_{2}-2 \rho}\|x\|^{2} .
$$

On the other hand, it can be readily checked that the range of the operator $\left(\left(S_{\alpha}-2 \rho I\right)^{-1}+\lambda A^{\alpha} V_{\rho \alpha}^{-1}(\lambda)\right)$ is all of $X$, by (4.20) and since the null space of its adjoint $\left(S_{\alpha}-2 \rho I\right)^{-1}+\bar{\lambda} A^{\alpha} V_{\rho \alpha}^{-1}(\bar{\lambda})$ is the trivial space. This together with (4.20) yields (4.19), hence (4.13). Finally, a uniform bound on $\mathscr{G}_{\rho \alpha}(\lambda)$ in $L(X)$ for $\operatorname{Re} \lambda>0$ follows from (3.2) of Proposition 3.2. Lemma 4.2 is proved. 
The proof of Proposition 4.1 is complete.

4.2. Proof of (4.3). We similarly compute by (4.5) and (4.7)

$$
\begin{aligned}
\lambda A^{1 / 2} & V_{\rho \alpha}^{-1}(\lambda)-\lambda A^{1 / 2} V_{B}^{-1}(\lambda) \\
& =\lambda A^{1 / 2} V_{\rho \alpha}^{-1}(\lambda)\left(B-2 \rho A^{\alpha}\right) \lambda V_{B}^{-1}(\lambda) \\
& =\lambda^{1 / 2} A^{1 / 2+\alpha / 2} V_{\rho \alpha}^{-1}(\lambda)\left(S_{\alpha}-2 \rho I\right) \lambda^{3 / 2} A^{\alpha / 2} V_{B}^{-1}(\lambda) .
\end{aligned}
$$

But $\lambda A^{1 / 2} V_{\rho \alpha}^{-1}(\lambda)$ and $\lambda^{1 / 2} A^{1 / 2+\alpha / 2} V_{\rho \alpha}^{-1}(\lambda)$ are uniformly bounded in $L(X)$ for all $\lambda$ with $\operatorname{Re} \lambda>0$, by (3.2) and (3.6) respectively. Thus, identity (4.21) shows that the desired bound (4.2) for $\lambda A^{1 / 2} V_{B}^{-1}(\lambda)$ holds true as soon as we prove that $\lambda^{3 / 2} A^{\alpha / 2} V_{B}^{-1}(\lambda)$ is uniformly bounded in $L(X)$ for all $\lambda$ with $\operatorname{Re} \lambda>0$. This is then the special case $\beta=0$ of the following more general result (which we shall use also in $\S 5$ below with $\alpha=\frac{1}{2}, \beta=-\alpha$ ).

Proposition 4.3. With $\frac{1}{2} \leq \alpha \leq 1$ given, let $-\alpha \leq \beta \leq \alpha$. Then, there exists a positive constant $k_{\alpha \beta}$ such that the following uniform bound holds true for all $\lambda$ with $\operatorname{Re} \lambda>0$ :

$$
\left\|\lambda^{3 / 2-\beta / 2 \alpha} A^{\alpha / 2} V_{B}^{-1}(\lambda) A^{\beta / 2}\right\|_{L(X)} \leq k_{\alpha \beta} .
$$

Proof of Proposition 4.3. By (4.5) and (4.7)

$$
\begin{aligned}
\lambda^{3 / 2-} & \beta / 2 \alpha \\
& A^{\alpha / 2} V_{\rho \alpha}^{-1}(\lambda) A^{\beta / 2}-\lambda^{3 / 2-\beta / 2 \alpha} A^{\alpha / 2} V_{B}^{-1}(\lambda) A^{\beta / 2} \\
& =\lambda^{3 / 2-\beta / 2 \alpha} A^{\alpha / 2} V_{\rho \alpha}^{-1}(\lambda)\left(B-2 \rho A^{\alpha}\right) \lambda V_{B}^{-1}(\lambda) A^{\beta / 2} \\
& =\lambda A^{\alpha} V_{\rho \alpha}^{-1}(\lambda)\left(S_{\alpha}-2 \rho I\right) \lambda^{3 / 2-\beta / 2 \alpha} A^{\alpha / 2} V_{B}^{-1}(\lambda) A^{\beta / 2}
\end{aligned}
$$

from which

$$
\begin{aligned}
& \lambda^{3 / 2-\beta / 2 \alpha} A^{\alpha / 2} V_{\rho \alpha}^{-1}(\lambda) A^{\beta / 2} \\
& \quad=\left[I+\lambda A^{\alpha} V_{\rho \alpha}^{-1}(\lambda)\left(S_{\alpha}-2 \rho I\right)\right] \lambda^{3 / 2-\beta / 2 \alpha} A^{\alpha / 2} V_{B}^{-1}(\lambda) A^{\beta / 2}
\end{aligned}
$$

counterpart of (4.11). By Lemma 4.2, the desired uniform bound (4.22) is achieved if and only if the left hand side of (4.24) is uniformly bounded in $L(X)$ for $\operatorname{Re} \lambda>0$. To establish this, since $(\alpha+\beta) / 2 \leq \alpha$ under the present assumption, we now invoke the uniform bound (3.5) with $\theta$ identified by $\alpha \theta=(\alpha+\beta) / 2$ so that

$$
\theta=\frac{1}{2}+\frac{\beta}{2 \alpha}, \quad 2-\theta=3 / 2-\frac{\beta}{2 \alpha}
$$

and our claim is proved. Thus, from (4.24) and (4.12)

$$
\lambda^{3 / 2-\beta / 2 \alpha} V_{B}^{-1}(\lambda) A^{\beta / 2}=\mathscr{G}_{\rho \alpha}^{-1}(\lambda) \lambda^{3 / 2-\beta / 2 \alpha} A^{\alpha / 2} V_{\rho \alpha}^{-1}(\lambda) A^{\beta / 2}
$$


and the uniform bounds (4.13) of Lemma 4.2 and (3.5) applied to (4.26) prove (4.22) as desired.

4.3. Proof of (4.4). Again by (4.5) and (4.7)

$$
\begin{gathered}
\lambda^{2} V_{\rho \alpha}^{-1}(\lambda)-\lambda^{2} V_{B}^{-1}(\lambda)=\lambda^{2} V_{\rho \alpha}^{-1}(\lambda)\left(B-2 \rho A^{\alpha}\right) \lambda V_{B}^{-1}(\lambda) \\
=\lambda^{3 / 2} A^{\alpha / 2} V_{\rho \alpha}^{-1}(\lambda)\left(S_{\alpha}-2 \rho I\right) \lambda^{3 / 2} A^{\alpha / 2} V_{B}^{-1}(\lambda)
\end{gathered}
$$

The desired conclusion (4.4) follows now from (4.26) by virtue of the uniform bounds (3.1) for $\lambda^{2} V_{\rho \alpha}^{-1}(\lambda),(3.7)$ for $\lambda^{3 / 2} A^{\alpha / 2} V_{\rho \alpha}^{-1}(\lambda)$ and (4.22) for $\lambda^{3 / 2} A^{\alpha / 2} V_{B}^{-1}(\lambda)$.

5. Case $\frac{1}{2} \leq \alpha \leq 1$. A second proof of Theorem 1.1. We recall that our goal is to establish the uniform inequalities (4.2), (4.3), and (4.4) in order to attain the uniform bound (4.1). Our second proof will be based on the following factorization of the function $V_{\rho \alpha}(\lambda)$ in (1.13), which we shall consider only for $\alpha=\frac{1}{2}$. Let $0<\theta<\pi / 2$ be fixed and select $\rho$ to be $\rho=\cos \theta$, so that $0<\rho<1$. We factor the function $V_{\rho \alpha}(\lambda)$ for $\alpha=\frac{1}{2}$ into two commuting factors as follows

$$
\lambda^{2} I+\lambda 2 \rho A^{1 / 2}+A=\left(\lambda e^{-i \theta}+A^{1 / 2}\right)\left(\lambda e^{i \theta}+A^{1 / 2}\right)
$$

where here and thereafter we drop the identity operator $I$ in connection with the scalars $\lambda e^{ \pm i \theta}$. By virtue of (5.1), we then re-write the functtion $V_{B}(\lambda)$ in (1.15) more conveniently as follows

$$
\begin{aligned}
& V_{B}(\lambda)=\lambda^{2} I+\lambda B+A \\
&=\left(\lambda^{2} I+\lambda 2 \rho A^{1 / 2}+A\right)+\lambda\left(B-2 \rho A^{1 / 2}\right) \\
&=\left(\lambda e^{-i \theta}+A^{1 / 2}\right)\left(\lambda e^{i \theta}+A^{1 / 2}\right)+\lambda\left(B-2 \rho A^{1 / 2}\right) \\
&=\left(\lambda e^{-i \theta}+A^{1 / 2}\right) \\
& \times\left[I+\lambda\left(\lambda e^{-i \theta}+A^{1 / 2}\right)^{-1}\left(B-2 \rho A^{1 / 2}\right)\left(\lambda e^{i \theta}+A^{1 / 2}\right)^{-1}\right] \\
& \times\left(\lambda e^{i \theta}+A^{1 / 2}\right) .
\end{aligned}
$$

Thus, setting for notational convenience

$$
U(\lambda, \theta)=\left(\lambda e^{-i \theta}+A^{1 / 2}\right)^{-1}\left(B-2 \rho A^{1 / 2}\right)\left(\lambda e^{i \theta}+A^{1 / 2}\right)^{-1}
$$

we obtain from $(5.2)-(5.3)$

$$
V_{B}^{-1}(\lambda)=\left(\lambda e^{i \theta}+A^{1 / 2}\right)^{-1}(I+\lambda U(\lambda, \theta))^{-1}\left(\lambda e^{-i \theta}+A^{1 / 2}\right)^{-1} .
$$

[Setting $T(\lambda, \theta)=A^{1 / 2}\left(\lambda e^{i \theta}+A^{1 / 2}\right)^{-1}$, then $U(\lambda, \theta)$ may be viewed as an extension of the operator $\left(\lambda e^{-i \theta}+A^{1 / 2}\right)^{-1}\left(\bar{\lambda} e^{-i \theta}+A^{1 / 2}\right) T^{*}(\lambda, \theta)\left(A^{-1 / 2} B A^{-1 / 2}-2 \rho A^{-1 / 2}\right) T(\lambda, \theta)$ 
which is well defined as a bounded operator on all of $X$.] Hence the functions in (4.2), (4.3), (4.4) are re-written now more conveniently as follows (using an obvious commutativity at the right of (5.5)):

$$
\begin{aligned}
& A^{1 / 2} V_{B}^{-1}(\lambda) A^{1 / 2} \\
& =A^{1 / 2}\left(\lambda e^{i \theta}+A^{1 / 2}\right)^{-1}(I+\lambda U(\lambda, \theta))^{-1} A^{1 / 2}\left(\lambda e^{-i \theta}+A^{1 / 2}\right)^{-1} \\
& \lambda A^{1 / 2} V_{B}^{-1}(\lambda) \\
& \quad=A^{1 / 2}\left(\lambda e^{i \theta}+A^{1 / 2}\right)^{-1}(I+\lambda U(\lambda, \theta))^{-1} \lambda\left(\lambda e^{-i \theta}+A^{1 / 2}\right)^{-1}
\end{aligned}
$$

(5.6) $\lambda A^{1 / 2} V_{B}^{-1}(\lambda)$

(5.7) $\lambda^{2} V_{B}^{-1}(\lambda)$

$$
=\lambda\left(\lambda e^{-i \theta}+A^{1 / 2}\right)^{-1}(I+\lambda U(\lambda, \theta))^{-1} \lambda\left(\lambda e^{-i \theta}+A^{1 / 2}\right)^{-1},
$$

respectively. Thus, in view of $(5.5)-(5.7)$, in order to prove the uniform bounds (4.2)-(4.4), it remains to prove the following Proposition.

Proposition 5.1. With $\frac{1}{2} \leq \alpha \leq 1$ and $\rho_{1}>0$ the constants in assumption $(\mathrm{H} .3)=(1.5)$, select $0<\theta<\pi / 2$ such that with $\rho=\cos \theta$ we have

$$
2 \rho\left\|A^{-(\alpha-1 / 2)}\right\|_{L(X)}<\rho_{1} .
$$

Then, the following uniform bounds hold true for all $\lambda$ with $\operatorname{Re} \lambda>0$ :

$$
\begin{gathered}
\left\|\lambda\left(\lambda e^{ \pm i \theta}+A^{1 / 2}\right)^{-1}\right\|_{L(X)} \leq c_{1 \theta}, \\
\left\|A^{1 / 2}\left(\lambda e^{ \pm i \theta}+A^{1 / 2}\right)^{-1}\right\|_{L(X)} \leq c_{2 \theta}, \\
\left\|(I+\lambda U(\lambda, \theta))^{-1}\right\|_{L(X)} \leq c_{3 \theta},
\end{gathered}
$$

where the positive constants $c_{1 \theta}, c_{2 \theta}$ and $c_{3 \theta}$ depend on $\theta$, but not on $\lambda$.

Proof of Proposition 5.1. Proof of (5.9)-(5.10). The negative selfadjoint operator $-A^{1 / 2}$ (with spectrum on the negative real axis) is the generator of a strongly continuous, analytic (self-adjoint) semigroup on $X$. Thus, its resolvent $R\left(\mu,-A^{1 / 2}\right)=\left(\mu I+A^{1 / 2}\right)^{-1}$ satisfies the standard Hille's uniform bound

$$
\left\|R\left(\mu,-A^{1 / 2}\right)\right\|_{L(X)} \leq \frac{\text { const }_{\phi}}{|\mu|}, \quad \mu \in \Sigma_{\phi}
$$

and hence also (since $A^{1 / 2} R\left(\mu,-A^{1 / 2}\right)=I-\mu R\left(\mu,-A^{1 / 2}\right)$ )

$$
\left\|A^{1 / 2} R\left(\mu,-A^{1 / 2}\right)\right\|_{L(X)} \leq \text { const }_{\phi}, \quad \mu \in \Sigma_{\phi}
$$


where $\phi$ is a fixed but arbitrary angle $\pi / 2<\phi<\pi$, and

$$
\Sigma_{\phi}=\{\lambda \in \mathbf{C}:|\arg \lambda|<\phi\} .
$$

Then, as $\lambda$ runs over the open right hand side $\operatorname{Re} \lambda>0$ of the complex plane, we see that $\mu=\lambda e^{ \pm i \theta} \in \Sigma_{\phi}$, with $\phi=\pi / 2+\theta+\varepsilon<\pi$, for $0<\theta<\pi / 2$, in which case (5.12) $-(5.13)$ prove (5.9) $-(5.10)$. (One can also prove (5.9)-(5.10) by using the orthonormal basis of eigenvectors of $A$ on $X$.)

Proof of (5.11). First of all, since $(U(\lambda, \theta))^{*}=U(\bar{\lambda}, \theta)$, we see from

$$
\left\|(I+\bar{\lambda} U(\bar{\lambda}, \theta))^{-1}\right\|=\left\|\left[(I+\lambda U(\lambda, \theta))^{-1}\right]^{*}\right\|=\left\|(I+\lambda U(\lambda, \theta))^{-1}\right\|
$$

that it suffices to prove $(5.11)$ for $\operatorname{Re} \lambda>0$ and $\operatorname{Im} \lambda \geq 0$. We shall accomplish this in several steps.

Step 1. We begin by noticing that for all $\lambda$ with $\operatorname{Re} \lambda>0$, the following uniform lower and upper bounds hold true:

$$
0<c_{\theta}<\left\|\left(\bar{\lambda} e^{i \theta}+A^{1 / 2}\right)\left(\lambda e^{i \theta}+A^{1 / 2}\right)^{-1}\right\|_{L(X)} \leq C_{\theta}
$$

for two positive constants $c_{\theta}<C_{\theta}<\infty$ depending on $\theta$ but independent of $\lambda$. (We shall actually use only the upper bound in (5.15)). Indeed, from the first resolvent equation [K.1, p. 173]

$$
\begin{aligned}
\left(\lambda e^{i \theta}\right. & \left.+A^{1 / 2}\right)^{-1}-\left(\bar{\lambda} e^{i \theta}+A^{1 / 2}\right)^{-1} \\
& =R\left(\lambda e^{i \theta},-A^{1 / 2}\right)-R\left(\bar{\lambda} e^{i \theta},-A^{1 / 2}\right) \\
& =e^{i \theta}(\bar{\lambda}-\lambda) R\left(\bar{\lambda} e^{i \theta},-A^{1 / 2}\right) R\left(\lambda e^{i \theta},-A^{1 / 2}\right)
\end{aligned}
$$

we obtain after applying $\left(\bar{\lambda} e^{i \theta}+A^{1 / 2}\right)$ to (5.16)

$$
\text { (5.17) }\left(\bar{\lambda} e^{i \theta}+A^{1 / 2}\right)\left(\lambda e^{i \theta}+A^{1 / 2}\right)^{-1}=I-2 i e^{i \theta}(\operatorname{Im} \lambda) R\left(\lambda e^{i \theta},-A^{1 / 2}\right)
$$

and the uniform upper bound in (5.15) follows from (5.17) via (5.12) with $\mu=\lambda e^{i \theta}$ as before. (To show the uniform lower bound in (5.15), we work with the inverse

$$
\left[\left(\bar{\lambda} e^{i \theta}+A^{1 / 2}\right)\left(\lambda e^{i \theta}+A^{1 / 2}\right)^{-1}\right]^{-1}=\left(\lambda e^{i \theta}+A^{1 / 2}\right)\left(\bar{\lambda} e^{i \theta}+A^{1 / 2}\right)
$$

and then apply the preceding analysis to show that the right hand side of (5.18) is uniformly bounded above for $\operatorname{Re} \lambda>0$ in $L(X)$.) Thus (5.15) is fully proved. A proof of (5.15) by eigenvector expansion may also be given.

Step 2. Define

$$
Q_{\rho \alpha}=A^{-\alpha / 2} B A^{-\alpha / 2}-2 \rho A^{-(\alpha-1 / 2)} \in L(X)
$$


so that, by assumptions $(\mathrm{H} .3)=(1.5)$ and (5.8) we have that $Q_{\rho \alpha}$ is a strictly positive self-adjoint, bounded operator on $X$; in fact for $\|x\|=1$ :

$$
\begin{aligned}
\left(Q_{\rho \alpha} x, x\right) & \geq \rho_{1}-2 \rho\left(A^{-(\alpha-1 / 2)} x, x\right) \\
& \geq \rho_{1}-2 \rho\left\|A^{-(\alpha-1 / 2)}\right\| \geq \rho_{0}>0
\end{aligned}
$$

i.e. $Q_{\rho \alpha} \geq \rho_{0} I$ for some constant $\rho_{0}>0$ since $\sup \left(A^{-(\alpha-1 / 2)} x, x\right)=$ $\left\|A^{-(\alpha-1 / 2)}\right\|$, where the sup of the self-adjoint bounded operator $A^{-(\alpha-1 / 2)}$ is taken over all $x$ with $\|x\|=1$.

Step 3. Using the upper bound in inequality (5.15) and the definition (5.3) for $U(\lambda, \theta)$ we compute for $x \in X$

$$
\begin{aligned}
& C_{\theta}\|(I+\lambda U(\lambda, \theta)) x\|\|x\| \\
& \geq \mid\|(I+\lambda U(\lambda, \theta)) x\|\left\|\left(\bar{\lambda} e^{i \theta}+A^{1 / 2}\right)\left(\lambda e^{i \theta}+A^{1 / 2}\right)^{-1} x\right\| \\
& \geq\left|\left((I+\lambda U(\lambda, \theta)) x,\left(\bar{\lambda} e^{i \theta}+A^{1 / 2}\right)\left(\lambda e^{i \theta}+A^{1 / 2}\right)^{-1} x\right)\right| \\
& =\mid\left(\left[I+\lambda\left(\lambda e^{-i \theta}+A^{1 / 2}\right)^{-1}\left(B-2 \rho A^{1 / 2}\right)\left(\lambda e^{i \theta}+A^{1 / 2}\right)^{-1}\right] x,\right. \\
& \left.\quad\left(\bar{\lambda} e^{i \theta}+A^{1 / 2}\right)\left(\lambda e^{i \theta}+A^{1 / 2}\right)^{-1} x\right) \mid .
\end{aligned}
$$

Thus, we simplify (5.21) by writing

$$
C_{\theta}\|(I+\lambda U(\lambda, \theta)) x\|\|x\| \geq\left|J_{1}(\lambda, \theta, x)+J_{2}(\lambda, \theta, x)\right|
$$

where we have set for $x \in X$

$$
J_{1}(\lambda, \theta, x)=\left(x,\left(\bar{\lambda} e^{i \theta}+A^{1 / 2}\right)\left(\lambda e^{i \theta}+A^{1 / 2}\right)^{-1} x\right),
$$

(5.24) $J_{2}(\lambda, \theta, x)$

$$
=\left(\lambda\left(B-2 \rho A^{1 / 2}\right)\left(\lambda e^{i \theta}+A^{1 / 2}\right)^{-1} x,\left(\lambda e^{i \theta}+A^{1 / 2}\right)^{-1} x\right) .
$$

That $J_{2}$ is well-defined on $X$ follows as in (5.28), (5.29) below.

Step 4. The following properties of $J_{1}(\lambda, \theta, x)$ are needed below

$$
\operatorname{Re} J_{1}(\lambda, \theta, x) \geq 0 \text { for } \operatorname{Re} \lambda \geq|\operatorname{Im} \lambda|,
$$

In fact, setting $\zeta(\lambda)=\left(\lambda e^{i \theta}+A^{1 / 2}\right)^{-1} x$ and hence $x=\left(\lambda e^{i \theta}+A^{1 / 2}\right) \zeta(\lambda)$, we obtain from (5.23) after moving $\left(\bar{\lambda} e^{i \theta}+A^{1 / 2}\right)$ to the left side of the inner product

$$
\text { (5.27) } \begin{aligned}
J_{1}(\lambda, \theta, x)= & \left(\left(\lambda e^{-i \theta}+A^{1 / 2}\right)\left(\lambda e^{i \theta}+A^{1 / 2}\right) \zeta(\lambda), \zeta(\lambda)\right) \\
= & \left(\left(\lambda^{2} I+\lambda 2 \rho A^{1 / 2}+A\right) \zeta(\lambda), \zeta(\lambda)\right) \quad(\text { by }(5.1)) \\
= & {\left[(\operatorname{Re} \lambda)^{2}-(\operatorname{Im} \lambda)^{2}\right]\|\zeta(\lambda)\|^{2} } \\
& +2 \rho(\operatorname{Re} \lambda)\left\|A^{1 / 4} \zeta(\lambda)\right\|^{2}+\left\|A^{1 / 2} \zeta(\lambda)\right\|^{2} \\
& +2 i(\operatorname{Im} \lambda)\left[\rho\left\|A^{1 / 4} \zeta(\lambda)\right\|^{2}+(\operatorname{Re} \lambda)\|\zeta(\lambda)\|^{2}\right]
\end{aligned}
$$


from which (5.25)-(5.26) follow at once. (One may show (5.25)(5.26) also by the explicit eigenvector expansion expression of $J_{1}$.)

Step 5. Using (5.19), we re-write $J_{2}(\lambda, \theta, x)$ in (5.24) as (5.28) $J_{2}(\lambda, \theta, x)=\lambda\left(Q_{\rho \alpha} y, y\right)=(\operatorname{Re} \lambda)\left(Q_{\rho \alpha} y, y\right)+i(\operatorname{Im} \lambda)\left(Q_{\rho \alpha} y, y\right)$ where we have set for $x \in X$ (5.29)

$$
y(\lambda, \theta, x)=y=A^{\alpha / 2}\left(\lambda e^{i \theta}+A^{1 / 2}\right)^{-1} x .
$$

Hence, from (5.20), (5.26) (or (5.27)) and (5.28) we obtain for $\operatorname{Re} \lambda>0$ :

$$
\left(\operatorname{Im} J_{1}\right)\left(\operatorname{Im} J_{2}\right) \geq 0
$$

where we have suppressed the arguments $\lambda, \theta, x$.

Step 6. We compute by (5.30) and (5.28):

$$
\begin{aligned}
& \left|J_{1}+J_{2}\right|^{2}=\left(\operatorname{Re} J_{1}+\operatorname{Re} J_{2}\right)^{2}+\left(\operatorname{Im} J_{1}+\operatorname{Im} J_{2}\right)^{2} \\
& \quad=\left|J_{1}\right|^{2}+\left|J_{2}\right|^{2}+2\left(\operatorname{Re} J_{1}\right)\left(\operatorname{Re} J_{2}\right)+2\left(\operatorname{Im} J_{1}\right)\left(\operatorname{Im} J_{2}\right) \\
& \geq\left|J_{1}\right|^{2}+\left|J_{2}\right|^{2}+2\left(\operatorname{Re} J_{1}\right)\left(\operatorname{Re} J_{2}\right) \quad(\text { by }(5.30)) .
\end{aligned}
$$

Then (5.31) leads to the following two cases

$$
\left|J_{1}+J_{2}\right|^{2} \geq \begin{cases}\left|J_{1}\right|^{2}+|\lambda|^{2}\left(Q_{\rho \alpha} y, y\right)^{2} & \text { for } \operatorname{Re} \lambda \geq|\operatorname{Im} \lambda|, \\ (1-\varepsilon)\left|J_{1}\right|^{2}+\left(2-\frac{1}{\varepsilon}\right) & (\operatorname{Im} \lambda)^{2}\left(Q_{\rho \alpha} y, y\right)^{2} \\ & \text { for } 0<\operatorname{Re} \lambda \leq|\operatorname{Im} \lambda|\end{cases}
$$

for any $\frac{1}{2}<\varepsilon<1$. Indeed, to obtain (5.32) from (5.31) we just recall (5.25), while to obtain (5.33) from (5.31) we use

$$
\begin{aligned}
2\left(\operatorname{Re} J_{1}\right)(\operatorname{Re} \lambda)\left(Q_{\rho \alpha} y, y\right) & \geq-\varepsilon\left(\operatorname{Re} J_{1}\right)^{2}-\frac{1}{\varepsilon}(\operatorname{Re} \lambda)^{2}\left(Q_{\rho}\right. \\
|\lambda|^{2}-\frac{1}{\varepsilon}(\operatorname{Re} \lambda)^{2} & =\left(1-\frac{1}{\varepsilon}\right)(\operatorname{Re} \lambda)^{2}+(\operatorname{Im} \lambda)^{2} \\
& \geq\left(1-\frac{1}{\varepsilon}\right)(\operatorname{Im} \lambda)^{2}+(\operatorname{Im} \lambda)^{2}
\end{aligned}
$$

since $(1-1 / \varepsilon)<0$ and $|\operatorname{Im} \lambda| \geq \operatorname{Re} \lambda>0$. We note that $0<(2-1 / \varepsilon)<$ 1 so that (5.32) and (5.33) can be combined into

$$
\left|J_{1}+J_{2}\right|^{2} \geq(1-\varepsilon)\left|J_{1}\right|^{2}+(2-1 / \varepsilon)(\operatorname{Im} \lambda)^{2}\left(Q_{\rho \alpha} y, y\right),
$$

for any preassigned $\frac{1}{2}<\varepsilon<1$.

Step 7. The following Lemma is then crucial. 
LeMma 5.2. There exists a suitable constant $\frac{1}{2}<\varepsilon_{1}<1$ (constructively found in the proof below) such that for all $x \in X$ and all $0<\theta<\pi / 2$ we have

$$
\begin{aligned}
& \left|J_{1}(\lambda, \theta, x)+J_{2}(\lambda, \theta, x)\right|^{2} \\
& \quad \geq\left(1-\varepsilon_{1}\right)\left|J_{1}\right|^{2}+\left(2-1 / \varepsilon_{1}\right)(\operatorname{Im} \lambda)^{2}\left(Q_{\rho \alpha} y, y\right), \quad \operatorname{Re} \lambda>0 \\
& \quad \geq\left(1-\varepsilon_{1}\right)|| x \|^{4} \text { for } \operatorname{Re} \lambda>0 \text { and } \operatorname{Im} \lambda \geq 0 .
\end{aligned}
$$

The proof of Lemma 5.2 will be given in Step 9 below.

Step 8. Assuming for the moment Lemma 5.2 and recalling (5.22) we obtain that: for all $x \in X$ and all $\lambda$ with $\operatorname{Re} \lambda>0$ and $\operatorname{Im} \lambda \geq 0$ the following uniform bound holds true:

$$
\|[I+\lambda U(\lambda, \theta)] x\|\|x\| \geq \frac{\left(1-\varepsilon_{1}\right)^{1 / 2}}{C_{\theta}}\|x\|^{2} .
$$

Thus, from (5.36), in order to conclude with the uniform bound (5.11) for $\operatorname{Re} \lambda>0$ and $\operatorname{Im} \lambda \geq 0$ as desired, we need to check that the range of $(I+\lambda U(\lambda, \theta))$ is all of $X$ (for $\operatorname{Re} \lambda>0$, in fact). This, in turn, is seen by (5.36) and by verifying that the null space of its adjoint (see (5.3))

$$
\begin{aligned}
& (I+\lambda U(\lambda, \theta))^{*} \\
& \quad=I+\bar{\lambda}\left(\bar{\lambda} e^{-i \theta}+A^{1 / 2}\right)^{-1}\left(B-2 \rho A^{1 / 2}\right)\left(\bar{\lambda} e^{i \theta}+A^{1 / 2}\right)^{-1}
\end{aligned}
$$

is the trivial subspace. Indeed, let $(I+\lambda U(\lambda, \theta))^{*} x=0$ for a fixed $\lambda$ with $\operatorname{Re} \lambda>0$ and thus, from (5.37), let

$$
\bar{\lambda}\left(\bar{\lambda} e^{-i \theta}+A^{1 / 2}\right)^{-1}\left(B-2 \rho A^{1 / 2}\right)\left(\bar{\lambda} e^{i \theta}+A^{1 / 2}\right)^{-1} x=-x
$$

with $x \in X$. We shall show that $x=0$. In fact, we first multiply (5.38) by the uniformly bounded operator

$$
\left(\lambda e^{-i \theta}+A^{1 / 2}\right)^{-1}\left(\bar{\lambda} e^{-i \theta}+A^{1 / 2}\right)
$$

(see (5.15)) and we next take the inner product with $x$. We obtain

$$
\begin{aligned}
\bar{\lambda}((B & \left.\left.-2 \rho A^{1 / 2}\right)\left(\bar{\lambda} e^{i \theta}+A^{1 / 2}\right)^{-1} x,\left(\bar{\lambda} e^{i \theta}+A^{1 / 2}\right)^{-1} x\right) \\
& =-\left(x,\left(\lambda e^{i \theta}+A^{1 / 2}\right)\left(\bar{\lambda} e^{i \theta}+A^{1 / 2}\right)^{-1} x\right)=-J_{1}(\bar{\lambda}, \theta, x)
\end{aligned}
$$

where in the last step we have recalled (5.23). Taking the imaginary part of identity $(5.39)$ and multiplying it by $(\operatorname{Im} \bar{\lambda})$ yields (since the inner product on the left of (5.39) is real):

(5.40) $(\operatorname{Im} \bar{\lambda})^{2}\left(\left(B-2 \rho A^{1 / 2}\right)\left(\bar{\lambda} e^{i \theta}+A^{1 / 2}\right)^{-1} x,\left(\bar{\lambda} e^{i \theta}+A^{1 / 2}\right)^{-1} x\right)$

$$
=-(\operatorname{Im} \bar{\lambda})\left(\operatorname{Im} J_{1}(\bar{\lambda}, \theta, x)\right) \text {. }
$$


But the left hand side of (5.40) is non-negative, while the right hand side of (5.40) is nonpositive by (5.26). Hence, each side of (5.40) is equal to zero. For $\operatorname{Im} \lambda \neq 0$, we then plainly obtain $\left(\bar{\lambda} e^{i \theta}+A^{1 / 2}\right)^{-1} x=$ 0 and hence $x=0$. For $\operatorname{Im} \lambda=0$, we take instead the real part in (5.39) and use now (5.25). By a similar argument, we again obtain $x=0$, as desired. Thus (5.11) holds true for $\operatorname{Re} \lambda>0$ and $\operatorname{Im} \lambda \geq 0$. But the observation at the beginning of the proof of (5.11) shows that this is enough to extend the validity of (5.11) to all of $\lambda$ with $\operatorname{Re} \lambda>0$. It remains to prove Lemma 5.2.

Step 9. Proof of Lemma 5.2. We must show (5.35).

(i) To this end, we recall the definition (5.23) of $J_{1}$ and identity (5.17) to obtain

$$
\text { (5.41) } \begin{aligned}
J_{1}(\lambda, \theta, x)= & \|x\|^{2}+2 i(\operatorname{Im} \lambda) e^{-i \theta}\left(x, R\left(\lambda e^{i \theta},-A^{1 / 2}\right) x\right) \\
= & \left\{\|x\|^{2}-2(\operatorname{Im} \lambda) \operatorname{Im}\left[e^{-i \theta}\left(x, R\left(\lambda e^{i \theta},-A^{1 / 2}\right) x\right]\right\}\right. \\
& +i 2(\operatorname{Im} \lambda) \operatorname{Re}\left[e^{-i \theta}\left(x, R\left(\lambda e^{i \theta},-A^{1 / 2}\right) x\right] .\right.
\end{aligned}
$$

Thus (5.41) implies

$$
\begin{aligned}
(5.42)\left|J_{1}(\lambda, \theta, x)\right|^{2}= & \|x\|^{4}+4(\operatorname{Im} \lambda)^{2}\left|e^{-i \theta}\left(x, R\left(\lambda e^{i \theta},-A^{1 / 2}\right) x\right)\right|^{2} \\
& -4\|x\|^{2}(\operatorname{Im} \lambda) \operatorname{Im}\left[e^{-i \theta}\left(x, R\left(\lambda e^{i \theta},-A^{1 / 2}\right) x\right]\right.
\end{aligned}
$$

where using the orthonormal basis $\left\{e_{n}\right\}$ of eigenvectors of $A$ with eigenvalues $\left\{\mu_{n}\right\}, 0<\mu_{1} \leq \mu_{2} \leq \cdots$, we have

$$
\begin{gathered}
e^{-i \theta}\left(x, R\left(\lambda e^{i \theta},-A^{1 / 2}\right) x\right)=e^{-i \theta}\left(x, \sum_{n} \frac{1}{\lambda e^{i \theta}+\mu_{n}^{1 / 2}}\left(x, e_{n}\right) e_{n}\right) \\
=\sum_{n} \frac{\left|\left(x, e_{n}\right)\right|^{2}}{\bar{\lambda}+\mu_{n}^{1 / 2} e^{i \theta}}=\sum_{n} \frac{\lambda+\mu_{n}^{1 / 2} e^{-i \theta}}{\left|\lambda+\mu_{n}^{1 / 2} e^{-i \theta}\right|^{2}}\left|\left(x, e_{n}\right)\right|^{2} .
\end{gathered}
$$

Taking the imaginary part of (5.43) and inserting it into (5.42), we finally obtain

$$
\begin{aligned}
\left|J_{1}(\lambda, \theta, x)\right|^{2}= & \|x\|^{4}+4(\operatorname{Im} \lambda)^{2}\left|e^{-i \theta}\left(x, R\left(\lambda e^{i \theta},-A^{1 / 2}\right) x\right)\right|^{2} \\
& +4\|x\|^{2}(\operatorname{Im} \lambda) \sum_{n} \frac{\mu_{n}^{1 / 2} \sin \theta}{\left|\lambda+\mu_{n}^{1 / 2} e^{-i \theta}\right|^{2}}\left|\left(x, e_{n}\right)\right|^{2} \\
& -4\|x\|^{2}(\operatorname{Im} \lambda)^{2} \sum_{n} \frac{\left|\left(x, e_{n}\right)\right|^{2}}{\left|\lambda+\mu_{n}^{1 / 2} e^{-i \theta}\right|^{2}} .
\end{aligned}
$$

We note that $(\operatorname{Im} \lambda) \sin \theta \geq 0$ under our present assumptions. 
(ii) Recalling the positivity property (5.20) for $Q_{\rho \alpha}$ and the definition (5.29) of $y$, we obtain with $\rho_{0}>0$ :

$$
\begin{aligned}
\left(Q_{\rho \alpha} y, y\right) & \geq \rho_{0}\|y\|^{2}=\rho_{0}\left\|A^{\alpha / 2}\left(\lambda e^{i \theta}+A^{1 / 2}\right)^{-1} x\right\|^{2} \\
& =\rho_{0} \sum_{n} \frac{\mu_{n}^{\alpha}}{\left|\lambda e^{i \theta}+\mu_{n}^{1 / 2}\right|^{2}}\left|\left(x, e_{n}\right)\right|^{2} \\
& =\rho_{0} \sum_{n} \frac{\mu_{n}^{\alpha}}{\left|\lambda+\mu_{n}^{1 / 2} e^{-i \theta}\right|^{2}}\left|\left(x, e_{n}\right)\right|^{2} .
\end{aligned}
$$

(iii) Plainly, it suffices to prove (5.35) for $x \in X$ with $\|x\|=1$. Thus, combining (5.44) with (5.45) we finally obtain taking $\frac{1}{2}<\varepsilon<1$ and $x \in X$ with $\|x\|=1$ :

$$
\begin{aligned}
& (1-\varepsilon)\left|J_{1}\right|^{2}+(2-1 / \varepsilon)(\operatorname{Im} \lambda)^{2}\left(Q_{\rho \alpha} y, y\right) \\
& \geq(1-\varepsilon)+4(1-\varepsilon)(\operatorname{Im} \lambda)^{2}\left|e^{-i \theta}\left(x, R\left(\lambda e^{i \theta},-A^{1 / 2}\right) x\right)\right|^{2} \\
& \quad+4(1-\varepsilon)(\operatorname{Im} \lambda) \sin \theta \sum_{n} \frac{\mu_{n}^{1 / 2}\left|\left(x, e_{n}\right)\right|^{2}}{\left|\lambda+\mu_{n}^{1 / 2} e^{-i \theta}\right|^{2}} \\
& \quad+(\operatorname{Im} \lambda)^{2} \sum_{n} \frac{f_{n \alpha}(\varepsilon)\left|\left(x, e_{n}\right)\right|^{2}}{\left|\lambda+\mu_{n}^{1 / 2} e^{-i \theta}\right|^{2}}
\end{aligned}
$$

where

$$
f_{n \alpha}(\varepsilon)=(2-1 / \varepsilon) \rho_{0} \mu_{n}^{\alpha}-4(1-\varepsilon), \quad \frac{1}{2}<\varepsilon<1 .
$$

Our task is now to show that we can select $\frac{1}{2}<\varepsilon_{1}<1$ such that

$$
f_{n \alpha}\left(\varepsilon_{1}\right) \geq 0 \text { for all } n
$$

after which, dropping non-negative terms in (5.46) we get for $\operatorname{Re} \lambda>0$ and $\operatorname{Im} \lambda \geq 0$ :

$$
\left(1-\varepsilon_{1}\right)\left|J_{1}(\lambda, \theta, x)\right|^{2}+\left(2-1 / \varepsilon_{1}\right)(\operatorname{Im} \lambda)^{2}\left(Q_{\rho \alpha} y, y\right) \geq 1-\varepsilon_{1}>0
$$

with $x \in X,\|x\|=1$, and Lemma 5.2 is proved.

But (5.48) can be easily checked since with the eigenvalues $\left\{\mu_{n}\right\}$, $0<\mu_{n}$, ordered in increasing fashion, we have

$$
f_{n \alpha}(\varepsilon) \geq f_{1 \alpha}(\varepsilon)=(2-1 / \varepsilon) \rho_{0} \mu_{1}^{\alpha}-4(1-\varepsilon), \quad \frac{1}{2}<\varepsilon<1
$$

while $f_{1 \alpha}(\varepsilon)$ is strictly increasing and $f_{1 \alpha}(\varepsilon=1)=\rho_{0} \mu_{1}^{\alpha}>0$. Indeed, we see directly that to get $f_{1 \alpha}(\varepsilon)>0$ we can take any $\varepsilon$ satisfying

$$
1>\varepsilon>\frac{-\left(\rho_{0} \mu_{1}^{\alpha}-2\right)+\sqrt{\left(\rho_{0} \mu_{1}^{\alpha}\right)+4}}{4}>\frac{1}{2}
$$

and (5.48) follows. 
6. Case $\alpha=\frac{1}{2}$. Proof of Theorem 1.2. The idea of the proof is based on the following three-step procedure ([L-T.1]). We show that the operator $\mathscr{A}_{B}$ generates a strongly continuous analytic semigroup of contractions (i) first on the larger space $Z=D\left(A^{1 / 4}\right) \times\left[D\left(A^{1 / 4}\right)\right]^{\prime}$ with norm (1.18)-(1.19), then on the smaller space $Y=D\left(A^{3 / 4}\right) \times D\left(A^{1 / 4}\right)$ with norm (1.18). We finally interpolate. More precisely, we apply the interpolation theorem [L-M.1, Theorem 5.1, p. 27] to, say, Hille's characterizations in $Z$ and $Y$ of $R\left(\lambda, \mathscr{A}_{B}\right)$. We thus obtain that for all $\lambda$ in the complement $\Sigma_{c}$ of a suitable triangular sector

$$
\Sigma=\left\{\lambda:|\arg \lambda|>\theta_{0}, \pi / 2<\theta_{0}<2 \pi\right\},
$$

the operator $R\left(\lambda, \mathscr{A}_{B}\right)$ is a continuous operator from the interpolation space

$$
\begin{aligned}
{[Y, Z]_{\theta} } & =\left[D\left(A^{3 / 4}\right) \times D\left(A^{1 / 4}\right), D\left(A^{1 / 4}\right) \times\left[D\left(A^{1 / 4}\right)\right]^{\prime}\right]_{\theta} \\
& =D\left(A^{3 / 4-\theta / 2}\right) \times D\left(A^{1 / 4-\theta / 2}\right)
\end{aligned}
$$

into itself and satisfies here Hille's characterization

$$
\left\|R\left(\lambda, \mathscr{A}_{B}\right)\right\|_{L\left([Y, Z]_{\theta}\right)} \leq \frac{\text { const }}{|\lambda|}, \quad \lambda \in \Sigma_{c}
$$

where in (6.1) we have used the conventional notation that $D\left(A^{-\beta}\right)$ for $\beta>0$ means $\left[D\left(A^{\beta}\right)\right]^{\prime}$, the dual space with respect to the $X$-topology.

Plainly, the strongly continuous semigroup of contractions generated by $\mathscr{A}_{B}$ on $E$ (see $(1.2($ iii) )) extends, respectively restricts, to $Z$, respectively $Y$, with these same properties. The issue of analyticity is dealt with in the next two subsections.

6.1. $\mathscr{A}_{B}$ generates an analytic semigroup on $Z=D\left(A^{1 / 4}\right) \times\left[D\left(A^{1 / 4}\right)\right]^{\prime}$. We shall establish that there exists a positive constant $C$ such that for all $\lambda$ with $\operatorname{Re} \lambda>0$ the following uniform bound holds

$$
\begin{aligned}
& \left\|\lambda R\left(\lambda, \mathscr{A}_{B}\right)\right\|_{L(Z)} \\
& =\left\|\left[\begin{array}{cc}
A^{1 / 4} & 0 \\
0 & A^{-1 / 4}
\end{array}\right]\left[\begin{array}{cc}
I-V_{B}^{-1}(\lambda) A & \lambda V_{B}^{-1}(\lambda) \\
-\lambda V_{B}^{-1}(\lambda) A & \lambda^{2} V_{B}^{-1}(\lambda)
\end{array}\right]\left[\begin{array}{cc}
A^{-1 / 4} & 0 \\
0 & A^{1 / 4}
\end{array}\right]\right\|_{L(W)} \leq C
\end{aligned}
$$

$W=X \times X$, see (1.18)-(1.19); equivalently, that there exists a positive constant $M$ such that for all $\lambda$ with $\operatorname{Re} \lambda>0$ we have:

$$
\begin{gathered}
\left\|A^{1 / 4} V_{B}^{-1}(\lambda) A^{3 / 4}\right\|_{L(X)} \leq M, \\
\left\|\lambda A^{1 / 4} V_{B}^{-1}(\lambda) A^{1 / 4}\right\|_{L(X)} \leq M, \\
\left\|\lambda^{2} A^{-1 / 4} V_{B}^{-1}(\lambda) A^{1 / 4}\right\|_{L(X)} \leq M .
\end{gathered}
$$


The proof of inequalities (6.4)-(6.6) can be accomplished by using the same techniques that were employed in $\S 4$ in the proof of inequalities (4.2)-(4.4). In fact, the task now is easier than in $\S 4$, in that we shall have no need now of the interpolating inequalities (3.4) and (3.5) (in the special case $\theta=\frac{1}{2}$ of $\left.(3.6)-(3.7)\right)$ used in $\S 4$, only of the original inequalities (3.1)-(3.3) (we recall that (3.1)-(3.3) collectively state the basic inequality (3.1) for $R\left(\lambda, \mathscr{A}_{\rho \alpha}\right)$ ). Throughout this section, we drop the subscript $\alpha=\frac{1}{2}$ and write $V_{\rho}^{-1}, S$, etc., instead of $V_{\rho \alpha}^{-1}(\lambda),(1.13)$, $S_{\alpha},(4.7)$, etc. Moreover, the two step procedure of $\S 4$ will reduce now to just invoking the fundamental Lemma 4.2.

To prove (6.4), (6.5), (6.6) we first establish the following identities, respectively

$$
\begin{aligned}
& A^{1 / 4} V_{\rho}^{-1}(\lambda) A^{3 / 4} \\
& \quad=\left[I+\lambda A^{1 / 2} V_{\rho}^{-1}(\lambda)(S-2 \rho I)\right] A^{1 / 4} V_{B}^{-1}(\lambda) A^{3 / 4}
\end{aligned}
$$

(6.8) $\lambda A^{1 / 4} V_{\rho}^{-1}(\lambda) A^{1 / 4}$

$$
=\left[I+\lambda A^{1 / 2} V_{\rho}^{-1}(\lambda)(S-2 \rho I)\right] \lambda A^{1 / 4} V_{B}^{-1}(\lambda) A^{1 / 4},
$$

(6.9) $\lambda^{2} A^{-1 / 4} V_{\rho}^{-1}(\lambda) A^{1 / 4}$

$$
=\lambda^{2} A^{-1 / 4} V_{B}^{-1}(\lambda) A^{1 / 4}\left[I+(S-2 \rho I) \lambda A^{1 / 2} V_{\rho}^{-1}(\lambda)\right] \text {. }
$$

Then, application of Lemma 4.2 with $\alpha=\frac{1}{2}$ to (6.7)-(6.9) together with properties (3.1)-(3.3) yields (6.4)-(6.6), respectively. Identities (6.8)-(6.9) are proved as in $\S 4$ by use of (4.5) and (4.7) with $\alpha=\frac{1}{2}$; instead, for inequality (6.9) we use $V_{\rho}^{-1}-V_{B}^{-1}=V_{B}^{-1}\left(V_{B}-V_{\rho}\right) V_{\rho}^{-1}$.

6.2. $\mathscr{A}_{B}$ generates an analytic semigroup on $Y=D\left(A^{3 / 4}\right) \times D\left(A^{1 / 4}\right)$.

Here we shall establish that there exists a positive constant $C$ such that for all $\lambda$ with $\operatorname{Re} \lambda>0$ the following uniform bound holds true

(6.10) $\left\|\lambda R\left(\lambda, \mathscr{A}_{B}\right)\right\|_{L(Y)}$

$$
=\left\|\left[\begin{array}{cc}
A^{3 / 4} & 0 \\
0 & A^{3 / 4}
\end{array}\right]\left[\begin{array}{cc}
I-V_{B}^{-1}(\lambda) A & \lambda V_{B}^{-1}(\lambda) \\
-\lambda V_{B}^{-1}(\lambda) A & \lambda^{2} V_{B}^{-1}(\lambda)
\end{array}\right]\left[\begin{array}{cc}
A^{-3 / 4} & 0 \\
0 & A^{-1 / 4}
\end{array}\right]\right\|_{L(W)} \leq C,
$$

$W=X \times X$, see (1.18); equivalently, that there exists a positive constant $M$ such that for all $\lambda$ with $\operatorname{Re} \lambda>0$ we have

$$
\begin{gathered}
\left\|A^{3 / 4} V_{B}^{-1}(\lambda) A^{1 / 4}\right\|_{L(X)} \leq M, \\
\left\|\lambda A^{3 / 4} V_{B}^{-1}(\lambda) A^{-1 / 4}\right\|_{L(X)} \leq M, \\
\left\|\lambda A^{1 / 4} V_{B}^{-1}(\lambda) A^{1 / 4}\right\|_{L(X)} \leq M, \\
\left\|\lambda^{2} A^{1 / 4} V_{B}^{-1}(\lambda) A^{-1 / 4}\right\|_{L(X)} \leq M .
\end{gathered}
$$


But we now note that inequalities (6.11), (6.13) and (6.14) are equivalent to the already established inequalities (6.4), (6.5), (6.6), respectively, simply by taking the adjoint and using $\left[V_{B}^{-1}(\lambda)\right]^{*}=V_{B}^{-1}(\bar{\lambda})$, see (1.17), and $\operatorname{Re} \lambda=\operatorname{Re} \bar{\lambda}$. To establish (6.12) we proceed as in $\S 4$. First, by (4.5) and (4.7) we obtain

$$
\begin{aligned}
\lambda A^{3 / 4} & V_{\rho}^{-1}(\lambda) A^{-1 / 4}-\lambda A^{3 / 4} V_{B}^{-1}(\lambda) A^{-1 / 4} \\
& =\lambda A^{3 / 4} V_{\rho}^{-1}(\lambda)\left(B-2 \rho A^{1 / 2}\right) \lambda V_{B}^{-1}(\lambda) A^{-1 / 4} \\
& =A V_{\rho}^{-1}(\lambda)(S-2 \rho I) \lambda^{2} A^{1 / 4} V_{B}^{-1}(\lambda) A^{-1 / 4}
\end{aligned}
$$

Applying now the uniform bounds (3.2) with $\alpha=\frac{1}{2},(3.3)$ and (4.22) of Proposition 4.3 with $\alpha=\frac{1}{2}=-\beta$ we obtain (6.12) from (6.15).

REMARK 6.1. In [C-T.1] we have provided an alternative proof which shows that $\mathscr{A}_{B}$ generates a strongly continuous analytic semigroup on the smaller space $Y$, as desired, simply as a consequence that $\mathscr{A}_{B}$ generates a strongly continuous analytic semigroup on the larger space $Z$. This proof is based on the equivalence between the two norms (see (1.18) for $Y$ ):

$$
\begin{gathered}
\|y\|_{D\left(\mathscr{A}_{B}\right)}=\left\|\mathscr{A}_{B} y\right\|_{Z} \text { and } \\
\mid y \|_{Y}=\left\{\left\|A^{3 / 4} y_{1}\right\|_{X}^{2}+\left\|A^{1 / 4} y_{2}\right\|_{X}^{2}\right\}^{1 / 2}
\end{gathered}
$$

for $y=\left(y_{1}, y_{2}\right) \in D\left(\mathscr{A}_{B}\right)$ which, as a linear space, coincide with $Y$ (recall that the point $\lambda=0$ is in the resolvent set of $\mathscr{A}_{B}$ ). This equivalence is in turn established by using the properties that $\mathscr{A}_{B} \mathscr{A}_{0}^{-1}$ and $\mathscr{A}_{0} \mathscr{A}_{B}^{-1}$ are both in $L(Z)$, where $\mathscr{A}_{0}=\mathscr{A}_{\rho=0}$ (see (1.11)). Thus, in this proof, no use is made of the interpolating inequalities (3.4)-(3.5) unlike the proof given above which uses Proposition 4.3, hence (3.5). We refer to [C-T.1] for further details. The interpolating technique of the present section permits us to give a very short proof of the local result in [C-R.1, Corollary 3.2] by applying standard perturbation theory on $Y$.

Appendix A: The case $B=2 \rho A^{\alpha}$. Spectral properties of the operator $\mathscr{A}_{\rho \alpha}, 0 \leq \alpha$. For $\frac{1}{2} \leq \alpha, \mathscr{A}_{\rho \alpha}$ generates a strongly continuous, analytic semigroup of contractions on $E=D\left(A^{1 / 2}\right) \times X$. The present appendix extends to the range $\frac{1}{2} \leq \alpha$ the treatment of [C-T.1, §2], which (prompted by results in [T.2], [L-T.1, Application 4.4] in the case $\alpha=0$ of viscous damping $B=2 \rho I$ ) referred only to the case $\alpha=\frac{1}{2}$. It is throughout assumed that $0<\rho<\infty$ unless otherwise noted. 
For the operator $A$ subject to assumption (H.1), let $\left\{\mu_{n}\right\}_{n=1}^{\infty}, 0<$ $\mu_{1}<\mu_{2}<\cdots$, be its eigenvalues and let $\left\{e_{n}\right\}_{n=1}^{\infty}$ be the corresponding eigenvectors subject to the normalization condition (A.6) below
(A.1)
$A e_{n}=\mu_{n} e_{n}$.

For simplicity of exposition, we assume that the $\mu_{n}$ 's are all simple. The following spectral properties of the operator $\mathscr{A}_{\rho \alpha}$ in (1.8) show, in particular, that $\mathscr{A}_{\rho \alpha}$ has a special structure. In the general case where $\rho^{2} \neq \mu_{n}^{1-2 \alpha}$ for all $n$ (in particular for $\alpha=\frac{1}{2}, \rho \neq 1$ ), $\mathscr{A}_{\rho \alpha}$ is the direct (non-orthogonal) sum of two (explicitly identified) normal operators on $E$. In the special case where $\alpha \neq \frac{1}{2}$ and $\rho^{2}=\mu_{n^{*}}^{1-2 \alpha}$ for a (unique) value $n=n^{*}$, then $\mathscr{A}_{\rho \alpha}$ has a third direct sum component, which possesses a Jordan cell structure on the two dimensional generalized eigenspace of $\mathscr{A}_{\rho \alpha}$ corresponding to $n=n^{*}$. Finally, in the case $\alpha=\frac{1}{2}$, $\rho=1, \mathscr{A}_{\rho \alpha}$ is the infinite sum of Jordan cell operators of the type described, for all values of $n$. But in the range $\frac{1}{2} \leq \alpha$ the eigenvalues $\lambda_{n}^{+,-}$of $\mathscr{A}_{\rho \alpha}$ are contained in a triangular sector

$$
\Sigma=\left\{\lambda:|\arg \lambda| \geq \pi / 2+\theta_{0}, 0<\theta_{0}<\pi / 2\right\}
$$

since $\left|\operatorname{Im} \lambda_{n}^{+,-}\right| /\left|\operatorname{Re} \lambda_{n}^{+,-}\right| \leq$const. Hence, the above spectral properties imply that, in this case $\frac{1}{2} \leq \alpha$, the strongly continuous semigroup of contractions of $\exp \left(\mathscr{A}_{\rho \alpha} t\right)$ generated by $\mathscr{A}_{\rho \alpha}$ on $E(\S 1.2(\mathrm{iii}))$ is, in addition, analytic (holomorphic) here. Moreover, $\exp \left(\mathscr{A}_{\rho \alpha} t\right)$ admits explicit spectral expansions on $E$. This way one shows, a fortiori, part (i) of Proposition 3.1. This then implies the uniform Hille's bound (3.8) for the resolvent $R\left(\lambda, \mathscr{A}_{\rho \alpha}\right)$ and hence (via (1.2)) the uniform bounds (3.1), (3.2), (3.3) of part (ii) (except for the explicit constants involved).

LEMMA A.1 (spectral properties of $\left.\mathscr{A}_{\rho \alpha}\right)$. Let $0 \leq \alpha$ and $0<\rho<\infty$ be given.

(I)(a) The eigenvalues $\left\{\lambda_{n}^{+,-}\right\}_{n=1}^{\infty}$ of the operator $\mathscr{A}_{\rho \alpha}$ in (1.8), which for $\alpha<1$ has compact resolvent on $E=D\left(A^{1 / 2}\right) \times X$, are the solutions of

$$
\lambda^{2}+2 \rho \mu_{n}^{\alpha} \lambda+\mu_{n}=0
$$

(so that $\lambda_{n}^{+}+\lambda_{n}^{-}=-2 \rho \mu_{n}^{\alpha} ; \lambda_{n}^{+} \lambda_{n}^{-}=\mu_{n}$ ) and are given by

$$
\lambda_{n}^{+,-}=\left(-\rho \pm \sqrt{\rho^{2}-\mu_{n}^{1-2 \alpha}}\right) \mu_{n}^{\alpha}
$$


[in particular, for $\alpha=\frac{1}{2}$ and $0<\rho<1$, the case used in [C-T.1]

$$
\left.\lambda_{n}^{+,-}=\mu_{n}^{1 / 2} e^{ \pm i \Psi}, \quad e^{ \pm i \Psi}=-\rho \pm i \sqrt{1-\rho^{2}}, \quad \pi / 2<\Psi<\pi\right] .
$$

Moreover

$$
\left|\frac{\operatorname{Im} \lambda_{n}^{+,-}}{\operatorname{Re} \lambda_{n}^{+,-}}\right| \leq \text {Const, if and only if } \frac{1}{2} \leq \alpha .
$$

For $\alpha>\frac{1}{2}$, or $\alpha=\frac{1}{2}$ and $\rho \geq 1$, then, $\lambda_{n}^{+,-}$are all negative for $n$ sufficiently large and $\lambda_{n}^{-} \downarrow-\infty$ monotonically; for $\frac{1}{2}<\alpha<1$, or $\alpha=\frac{1}{2}$ and $\rho \geq 1$, then $\lambda_{n}^{+} \downarrow-\infty$ monotonically; for $\alpha=1$, then $\lambda_{n}^{+} \uparrow-\frac{1}{2} \rho$ monotonically; finally for $\alpha>1$, then $\lambda_{n}^{+} \uparrow 0$ monotonically. Thus the spectrum $\sigma\left(\mathscr{A}_{\rho \alpha}\right)$ of $\mathscr{A}_{\rho \alpha}$ is only point spectrum for $\alpha<1$ but contains the point $\lambda=-\frac{1}{2} \rho$ for $\alpha=1$, or the point $\lambda=0$ for $\alpha>1$, in its continuous spectrum. Thus $\mathscr{A}_{\rho \alpha}$ has compact resolvent for $\alpha<1$, but not for $\alpha \geq 1$.

(I)(b) The corresponding normalized eigenvectors $\left\{\Phi_{n}^{+,-}\right\}_{n=1}^{\infty}$ on $E$ are

$$
\boldsymbol{\Phi}_{n}^{+}=\left[\begin{array}{c}
e_{n} \\
\lambda_{n}^{+} e_{n}
\end{array}\right] ; \quad \Phi_{n}^{-}=k_{n}\left[\begin{array}{c}
e_{n} \\
\lambda_{n}^{-} e_{n}
\end{array}\right]
$$

in which case

$$
\left\|\Phi_{n}^{+,-}\right\|_{E}=1 \Leftrightarrow\left(\mu_{n}+\left|\lambda_{n}^{+}\right|^{2}\right)\left\|e_{n}\right\|^{2}=1, \quad k_{n}^{2}=\frac{\mu_{n}+\left|\lambda_{n}^{+}\right|^{2}}{\mu_{n}+\left|\lambda_{n}^{-}\right|^{2}}
$$

where

$$
\left\{\begin{array}{l}
\mu_{n}+\left|\lambda_{n}^{+,-}\right|^{2}=2 \mu_{n}, \quad \text { if } \rho^{2} \leq \mu_{n}^{1-2 \alpha}, \\
\mu_{n}+\left|\lambda_{n}^{+,-}\right|^{2}=\mu_{n}^{2 \alpha}\left(2 \rho^{2} \mp 2 \rho \sqrt{\rho^{2}-\mu_{n}^{1-2 \alpha}}\right),
\end{array}\right.
$$

$$
\text { if } \rho^{2}>\mu_{n}^{1-2 \alpha} \text {. }
$$

(II) The eigenvectors possess the following properties.

(A.9) $\left\{\Phi_{n}^{+}\right\}_{n=1}^{\infty}$ is an orthonormal family of eigenvectors on $E$,

(A.10) $\left\{\Phi_{n}^{-}\right\}_{n=1}^{\infty}$ is an orthonormal family of eigenvectors on $E$,

$$
\text { (A.11) } \quad\left(\Phi_{m}^{+}, \Phi_{n}^{-}\right)_{E}= \begin{cases}0, & n \neq m, \\ k_{n}\left(\mu_{n}+\lambda_{n}^{+} \bar{\lambda}_{n}^{-}\right)\left\|e_{n}\right\|_{X}^{2} & n=m ; \lambda_{n}^{+} \neq \lambda_{n}^{-}, \\ 1, & n=m, \lambda_{n}^{+}=\lambda_{n}^{-}\end{cases}
$$


(iii) (Completeness of eigenvectors of $\mathscr{A}_{\rho \alpha}$ on $E$ ). Let $\rho^{2} \neq \mu_{n}^{1-2 \alpha}$ for all $n$ so that $\lambda_{n}^{+} \neq \lambda_{n}^{-}$for all $n$. Then

$$
\overline{\operatorname{span}}\left\{\Phi_{n}^{+,-}\right\}_{n=1}^{\infty}=E .
$$

(iv) (Completeness of generalized eigenvectors of $\mathscr{A}_{\rho \alpha}$ on E) (a) First, let $\alpha \neq \frac{1}{2}$ and assume that $\rho$ satisfies

$$
\rho=\mu_{n^{*}}^{1-2 \alpha}
$$

for a positive integer $n=n^{*}$ (therefore unique), so that

$$
\lambda_{n^{*}}^{+}=\lambda_{n^{*}}^{-}=-\rho \mu_{n^{*}}^{\alpha}
$$

Define the vector $\Psi_{n^{*}}^{-}$by

$$
\Psi_{n^{*}}^{-}=\left[\begin{array}{c}
0 \\
e_{n^{*}}
\end{array}\right]
$$

so that

$$
\text { (A.16) } \quad \mathscr{A}_{\rho \alpha} \Psi_{n^{*}}^{-}=\lambda_{n^{*}}^{+} \Psi_{n^{*}}^{-}+\Phi_{n^{*}}^{+} ; \quad\left(\mathscr{A}_{\rho \alpha}-\lambda_{n^{*}}^{+} I\right)^{2} \Psi_{n^{*}}^{-}=0
$$

and $\Psi_{n^{*}}^{-}$is a generalized eigenvector of $\mathscr{A}_{\rho \alpha}$ corresponding to the double eigenvalue $\lambda_{n^{*}}^{+}$.

Then

$$
\overline{\operatorname{span}}\left\{\Phi_{n}^{+,-}, \Psi_{n^{*}}^{-}\right\}_{n=1}^{\infty}=E .
$$

(b) Next, let $\alpha=\frac{1}{2}$ and $\rho=1$ so that $\lambda_{n}^{+}=\lambda_{n}^{-}=-\mu_{n}^{1 / 2}$ for all $n$. Define the vector $\Psi_{n}^{-}$as in (A.15) for all $n$ so that now (A.16) holds for all $n$. Then

$$
\overline{\operatorname{span}}\left\{\Phi_{n}^{+}, \Psi_{n}^{-}\right\}_{n=1}^{\infty}=E
$$

where we note that

$$
\left(\Phi_{m}^{+}, \Psi_{n}^{-}\right)_{E}= \begin{cases}0, & n \neq m \\ \lambda_{n}^{+}\left\|e_{n}\right\|_{X}^{2} \neq 0, & n=m\end{cases}
$$

(A.20) $\left\{\sqrt{\mu_{n}+\left|\lambda_{n}^{+}\right|^{2}} \Psi_{n}^{-}\right\}_{n=1}^{\infty}$

is an orthonormal family of generalized eigenvectors on $E$.

(v) a) If $\rho^{2} \neq \mu_{n}^{1-2 \alpha}$ for all $n$ (so that $\lambda_{n}^{+} \neq \lambda_{n}^{-}$for all $n$ ), set

$$
E^{+}=\overline{\operatorname{span}}\left\{\Phi_{n}^{+}\right\}_{n=1}^{\infty} ; \quad E^{-}=\overline{\operatorname{span}}\left\{\Phi_{n}^{-}\right\}_{n=1}^{\infty} .
$$

Then, in this case

(A.22) $E=E^{+} \oplus E^{-} ; \quad E^{+} \cap E^{-}=\{0\}$

(direct, non-orthogonal sum). 
(b) If $\alpha \neq \frac{1}{2}$ and (A.13) holds, set

$$
\begin{gathered}
E_{n^{*}}^{+}=\overline{\operatorname{span}}\left\{\Phi_{n}^{+}\right\}_{n=1, n \neq n^{*}}^{\infty} ; \\
E_{n^{*}}^{-}=\overline{\operatorname{span}}\left\{\Phi_{n}^{-}\right\}_{n=1, n \neq n^{*}}^{\infty} ; \quad E_{n^{*}}=\operatorname{span}\left\{\Phi_{n^{*}}^{+}, \Psi_{n^{*}}^{-}\right\} .
\end{gathered}
$$

Then, in this case

(A.24) $E=E_{n^{*}}^{+} \oplus E_{n^{*}}^{-} \oplus E_{n^{*}}$;

$$
E_{n^{*}}^{+} \cap E_{n^{*}}^{-}=E_{n^{*}}^{+} \cap E_{n^{*}}=E_{n^{*}}^{-} \cap E_{n^{*}}=\{0\}
$$

(direct, non-orthogonal sum).

(c) If $\alpha=\frac{1}{2}$ and $\rho=1$, set $E^{+}$as in (A.21) and

$$
E_{\Psi}^{-}=\overline{\operatorname{span}}\left\{\Psi_{n}^{-}\right\}_{n=1}^{\infty} \text {. }
$$

Then, in this case

$$
\text { (A.26) } E=E^{+} \oplus E_{\Psi}^{-} ; \quad E^{+} \cap E_{\Psi}^{-}=\{0\}
$$

(direct, non-orthogonal sum).

(iv) From (A.9), (A.10), (A.20) it follows that [S.1, p. 250]: the operator $\mathscr{A}_{\rho \alpha}$ restricted over $E^{+}$, or $E^{-}$, is a normal operator (however, $\mathscr{A}_{\rho \alpha}$ is not normal on $E$ for $0<\rho$; however, $\mathscr{A}_{\rho \alpha}$ is a spectral operator in the sense of [D.1], [D-S.1]). The restriction $\mathscr{A}_{\rho \alpha}^{-}$of $\mathscr{A}_{\rho \alpha}$ over $E^{-}$ has compact resolvent. The restriction $\mathscr{A}_{\rho \alpha}^{+}$of $\mathscr{A}_{\rho \alpha}$ over $E^{+}$has compact resolvent for $\alpha<1$, but not for $\alpha \geq 1$. For $\alpha=1$, the operator $\left(\mathscr{A}_{\rho \alpha}^{+}\right)^{-1}+$ $2 \rho I$ is both normal and compact.

Proof. Direct verification of the above statements is left to the reader.

The above Lemma A.1 then provides spectral expansions for $x \in E$; for $\mathscr{A}_{\rho \alpha} x$ with $x \in D\left(\mathscr{A}_{\rho \alpha}\right)$; for $\exp \left(\mathscr{A}_{\rho \alpha} t\right) x$; for $R\left(\lambda, \mathscr{A}_{\rho \alpha}\right) x$; etc. In particular, as remarked above Lemma A.1, the expansion of the semigroup $\exp \left(\mathscr{A}_{\rho \alpha} t\right) x$ in the range $\frac{1}{2} \leq \alpha$ (where (A.4) holds and the eigenvalues $\lambda_{n}^{+,-}$are in the triangular sector $\Sigma$ defined above Lemma A.1) permits one to read off that $\exp \left(\mathscr{A}_{\rho \alpha} t\right)$ is analytic on $E$ on a suitable sector containing the positive real axis. For the sake of brevity, we confine ourselves here to carry out our further analysis only in the case (v)(a) where $\rho^{2} \neq \mu_{n}^{1-2 \alpha}$ for all $n$ (i.e. $\lambda_{n}^{+} \neq \lambda_{n}^{-}$for all $n$ ) where there is no Jordan component of $\mathscr{A}_{\rho \alpha}$, and leave the exceptional cases (v)(b) and $(\mathrm{v})(\mathrm{c})$ to the reader for the standard modifications which are required to handle the Jordan components of $\mathscr{A}_{\rho \alpha}$. For the purposes of $\S \S 3,4,5,6$, restriction to the case $(\mathrm{v})(\mathrm{a})$ with $\rho^{2} \neq \mu_{n}^{1-2 \alpha}$ for all $n$ is 
fully sufficient, since in the proofs of $\S \S 4-6$, we only need analyticity of $\exp \left(\mathscr{A}_{\rho \alpha} t\right)$ for $\frac{1}{2} \leq \alpha \leq 1$ and for just one value of $0<\rho$ sufficiently small.

LEMMA A.2. Let $0 \leq \alpha \leq 1$ and $0<\rho<\infty$ be given satisfying $\rho^{2} \neq \mu_{n}^{1-2 \alpha}$ for all $n, \quad$ so that $\lambda_{n}^{+} \neq \lambda_{n}^{-}$for all $n$

and case (v)(a) in Lemma A.1 above applies. As a consequence, for every $x \in E$, we have

$$
x=x^{+}+x^{-}, \quad x^{+} \in E^{+}, \quad x^{-} \in E^{-},
$$

$$
x^{+}=\sum_{n=1}^{\infty}\left(x^{+}, \boldsymbol{\Phi}_{n}^{+}\right)_{E} \boldsymbol{\Phi}_{n}^{+} ; \quad x^{-}=\sum_{n=1}^{\infty}\left(x^{-}, \boldsymbol{\Phi}_{n}^{-}\right)_{E} \boldsymbol{\Phi}_{n}^{-} .
$$

Let now $\mathscr{A}_{\rho \alpha}^{*}$ denote the adjoint operator of $\mathscr{A}_{\rho \alpha}$ in $E$ :

(II)

$$
\left(\mathscr{A}_{\rho \alpha} x, y\right)_{E}=\left(x, \mathscr{A}_{\rho \alpha}^{*} y\right)_{E}, \quad x \in D\left(\mathscr{A}_{\rho \alpha}\right), \quad y \in D\left(\mathscr{A}_{\rho \alpha}^{*}\right) . \quad \text { Then }
$$

(a):

$$
\begin{gathered}
\text { (A.30) } \mathscr{A}_{\rho \alpha}^{*}=\left[\begin{array}{cc}
0 & -I \\
A & -2 \rho A^{\alpha}
\end{array}\right] ; \quad \mathscr{A}_{\rho \alpha}^{*} \mathscr{A}_{\rho \alpha}-\mathscr{A}_{\rho \alpha} \mathscr{A}_{\rho \alpha}^{*}=4 \rho\left[\begin{array}{cc}
0 & A^{\alpha} \\
A^{1+\alpha} & 0
\end{array}\right], \\
D\left(\mathscr{A}_{\rho \alpha}\right)=D\left(\mathscr{A}_{\rho \alpha}^{*}\right)=D(A) \times D\left(A^{\alpha}\right)
\end{gathered}
$$

[so that $\mathscr{A}_{\rho \alpha}^{*}$ is normal if and only if $\rho=0$, the undamped case].

(b): The eigenvalues of $\mathscr{A}_{\rho \alpha}^{*}$ are given by $\overline{\lambda_{m}^{+,-}}$, with corresponding (normalized) eigenvectors

$$
\left[\begin{array}{c}
e_{m} \\
-\overline{\lambda_{m}^{+}} e_{m}
\end{array}\right], \quad k_{m}\left[\begin{array}{c}
e_{m} \\
-\overline{\lambda_{m}^{-}} e_{m}
\end{array}\right]
$$

(c): If we set

$$
v_{m}^{+}=\frac{\mu_{m}-\left(\overline{\lambda_{m}^{-}}\right)^{2}}{\mu_{m}+\left|\lambda_{m}^{-}\right|^{2}} ; \quad v_{m}^{-}=\frac{\mu_{m}-\left(\overline{\lambda_{m}^{+}}\right)^{2}}{\mu_{m}+\left|\lambda_{m}^{+}\right|^{2}}
$$

so that in the case $\alpha=\frac{1}{2}, 0<\rho<1$ of [C-T.1] we have

$$
v_{m}^{+}=\frac{\lambda_{m}^{-}-\lambda_{m}^{+}}{2 \lambda_{m}^{-}} ; \quad v_{m}^{-}=\overline{v_{m}^{+}}=\frac{\lambda_{m}^{+}-\lambda_{m}^{-}}{2 \lambda_{m}^{+}}
$$


then the following non-normalized eigenvectors of $\mathscr{A}_{\rho \alpha}^{*}$,

$$
\Phi_{m}^{*-}=\frac{1}{v_{m}^{-}}\left[\begin{array}{c}
e_{m} \\
-\overline{\lambda_{m}^{+}} e_{m}
\end{array}\right], \quad \Phi_{m}^{*+}=\frac{1}{v_{m}^{+}}\left[\begin{array}{c}
e_{m} \\
-\overline{\lambda_{m}^{-}} e_{m}
\end{array}\right]
$$

corresponding to the eigenvalues

$$
\overline{\lambda_{m}^{+}} \text {and } \overline{\lambda_{m}^{-}}
$$

respectively, form a bi-orthogonal system with respect to the eigenvectors $\left\{\Phi_{m}^{+,-}\right\}$of $\mathscr{A}_{\rho \alpha}$, corresponding to its eigenvalues $\lambda_{m}^{+,-}$:

$$
\left(\Phi_{m}^{*-}, \Phi_{n}^{+}\right)_{E}=\left(\Phi_{m}^{*+}, \Phi_{n}^{-}\right)_{E}=\operatorname{Kroneker} \delta_{m n}
$$

$$
\left(\boldsymbol{\Phi}_{m}^{*+}, \boldsymbol{\Phi}_{n}^{+}\right)_{E}=\left(\boldsymbol{\Phi}_{m}^{*-}, \boldsymbol{\Phi}_{n}^{-}\right)_{E}=0, \quad \forall n, m
$$

(d): Taking the E-inner product of $x$ given by (A.29) with $\Phi_{m}^{*+,-}$ and using properties (A.34)-(A.35) we obtain

$$
\left(x, \boldsymbol{\Phi}_{m}^{*-}\right)_{E}=\left(x^{+}, \boldsymbol{\Phi}_{m}^{+}\right)_{E} ; \quad\left(x, \boldsymbol{\Phi}_{m}^{*+}\right)_{E}=\left(x^{-}, \boldsymbol{\Phi}_{m}^{-}\right)_{E}
$$

whereby the expansion (A.29) for $x \in E$ becomes

$$
x=\sum_{n=1}^{\infty}\left(x, \boldsymbol{\Phi}_{n}^{*-}\right)_{E} \boldsymbol{\Phi}_{n}^{+}+\sum_{n=1}^{\infty}\left(x, \boldsymbol{\Phi}_{n}^{*+}\right)_{E} \boldsymbol{\Phi}_{n}^{-},
$$

more convenient than (A.28)-(A.29).

Proof. Direct verification is left to the reader.

From the space decomposition (A.22) and expansion (A.37) we obtain, in particular, an explicit representation for $\mathscr{A}_{\rho \alpha}$ and the corresponding strongly continuous semigroup $\exp \left(\mathscr{A}_{\rho \alpha} t\right)$. The latter shows, by inspection, that $\exp \left(\mathscr{A}_{\rho \alpha} t\right)$ is, in fact, analytic on $E$ in a suitable triangular sector around the positive real axis $R^{+}$.

Theorem A.3. Let $\frac{1}{2} \leq \alpha$ and $0<\rho<\infty$ be given satisfying assumption (A.27) of Lemma A.2. Then

(i) The operator $\mathscr{A}_{\rho \alpha}$ in (1.8) is the direct sum of two normal operators $\mathscr{A}_{\rho \alpha}^{+}$and $\mathscr{A}_{\rho \alpha}^{-}$defined as restrictions of $\mathscr{A}_{\rho \alpha}$ on $E^{+}$, and, respectively, on $E^{-}$, see Lemma A.1(vi). (A fortiori, $\mathscr{A}_{\rho \alpha}$ is similar to a normal operator on E, by virtue of Wermer theorem [D-S.1, III p. 1947]. Explicit construction of the corresponding similarity transformation can be obtained along the lines of the construction given in [T.2], [L-T.1, 
Application 4.4] with the identity operator I in these references replaced now by $A^{\alpha}$ ).

(ii) The expansion for $\mathscr{A}_{\rho \alpha}$ is given by (see A.37):

$$
\mathscr{A}_{\rho \alpha} x=\sum_{n=1}^{\infty} \lambda_{n}^{+}\left(x, \boldsymbol{\Phi}_{n}^{*-}\right)_{E} \boldsymbol{\Phi}_{n}^{+}+\sum_{n=1}^{\infty} \lambda_{n}^{-}\left(x, \boldsymbol{\Phi}_{n}^{*+}\right)_{E} \boldsymbol{\Phi}_{n}^{-},
$$

(iii) $\mathscr{A}_{\rho \alpha}$ generates a strongly continuous semigroup $\exp \left(\mathscr{A}_{\rho \alpha} t\right)$ on $E$ given explicitly by

$$
\begin{aligned}
\exp \left(\mathscr{A}_{\rho \alpha} t\right) x= & \sum_{n=1}^{\infty} \exp \left(\lambda_{n}^{+} t\right)\left(x, \Phi_{n}^{*-}\right)_{E} \Phi_{n}^{+} \\
& +\sum_{n=1}^{\infty} \exp \left(\lambda_{n}^{-} t\right)\left(x, \Phi_{n}^{*+}\right)_{E} \Phi_{n}^{-}
\end{aligned}
$$

which is analytic for $t>0$ or, more generally, on $\Sigma_{\theta_{0}}=\{\lambda:|\arg \lambda|<$ $\left.\theta_{0}\right\}$, where $\theta_{0}$ is defined above Lemma A.1. Moreover, as observed in $\S 1.2(\mathrm{iii}), \exp \left(\mathscr{A}_{\rho \alpha} t\right)$ is contraction on $E$.

REMARK A.1. Even in the case $\alpha=\frac{1}{2}$, the results of the present Appendix strengthen and refine those in [C-R.1], by providing more precise information about the spectral structure of $\mathscr{A}_{\rho \alpha}$. In particular, in the case $\alpha=\frac{1}{2}$ and $0<\rho<1$ [C-R.1] asserts only that $\mathscr{A}_{\rho \alpha}$ is similar to a normal operator, while Theorem A.3 above specifies that, in fact, $\mathscr{A}_{\rho \alpha}$ is the direct sum of two normal operators, a plainly more precise conclusion. As a consequence, the eigenvectors of $\mathscr{A}_{\rho \alpha}$ are only asserted in [C-R.1] to form a Riesz basis, in the case $\alpha=\frac{1}{2}$ and $0<\rho<1$, a plainly weaker conclusion than the precise spectral description and decompositions of Lemma A.1-A.2.

REMARK A.2. Expansions (A.38) and (A.39) show directly that for $0<\alpha<\frac{1}{2}$ the semigroup $\exp \left(\mathscr{A}_{\rho \alpha} t\right)$ is differentiable on $E$ for $t>0$; $\mathscr{A}_{\rho \alpha} \exp \left(\mathscr{A}_{\rho \alpha} t\right)$ is well defined on all of $E$.

Appendix B: Direct proof of inequalities (3.1) and (3.3) via eigenvector expansion. By using the orthonormal basis of eigenvectors of $A$ on $X$ with corresponding eigenvectors $\left\{\mu_{n}\right\}_{n=1}^{\infty}, 0<\mu_{1} \leq \mu_{2} \leq \cdots$, we easily see that the uniform bounds (3.1) and (3.3) are a consequence of the following Lemma (after replacing $\mu$ in the Lemma with $\mu_{n}$ ). 
LemMA B.1. Let $\rho>0$ and $\alpha \geq \frac{1}{2}$ be given. Let $\mu$ be a positive number satisfying $\mu \geq \mu_{1}>0$. Let $\beta$ denote either 0 or else 1 . Then the following uniform bound holds true for all $\lambda$ with $\operatorname{Re} \lambda>0$ :

(B.1) $\left|\frac{\lambda^{2}+2 \rho \lambda \mu^{\alpha}+\mu}{\lambda^{2(1-\beta)} \mu^{\beta}}\right|^{2} \geq C_{\rho \alpha \mu_{1}}$

$$
= \begin{cases}1 & \text { if } 2 \rho^{2} \mu_{1}^{2 \alpha-1} \geq 1, \\ 4 \rho^{2} \mu_{1}^{2 \alpha-1}\left(1-\rho^{2} \mu_{1}^{2 \alpha-1}\right) & \text { if } 2 \rho^{2} \mu_{1}^{2 \alpha-1}<1 .\end{cases}
$$

Proof. We set

(B.2) $\frac{\lambda}{\sqrt{\mu}}=\gamma e^{i \theta}$, so that for $\operatorname{Re} \lambda>0$ we have $\gamma>0,-\frac{\pi}{2}<\theta<\frac{\pi}{2}$.

We then compute

(B.3)

$$
\begin{aligned}
& \left|\frac{\lambda^{2}+2 \rho \lambda \mu^{\alpha}+\mu}{\lambda^{2(1-\beta)} \mu^{\beta}}\right|^{2} \\
& =\left|\frac{\lambda}{\sqrt{\mu}}\right|^{2(2 \beta-1)}\left|\frac{\lambda}{\sqrt{\mu}}+2 \rho \mu^{\alpha-1 / 2}+\frac{\sqrt{\mu}}{\lambda}\right|^{2} \\
& =\gamma^{2(2 \beta-1)}\left|\gamma(\cos \theta+i \sin \theta)+2 \rho \mu^{\alpha-1 / 2}+\frac{\cos \theta-i \sin \theta}{\gamma}\right|^{2} \\
& =\gamma^{2(2 \beta-1)}\left|\left[\left(\gamma+\frac{1}{\gamma}\right) \cos \theta+2 \rho \mu^{\alpha-1 / 2}\right]+i\left[\left(\gamma-\frac{1}{\gamma}\right) \sin \theta\right]\right|^{2} \\
& =\gamma^{2(2 \beta-1)}\left[\left(\gamma-\frac{1}{\gamma}\right)^{2}+4 \rho^{2} \mu^{2 \alpha-1}+4 \rho \mu^{\alpha-1 / 2}\left(\gamma+\frac{1}{\gamma}\right) \cos \theta+4 \cos ^{2} \theta\right]
\end{aligned}
$$

(dropping positive terms)

$$
\begin{aligned}
& \leq \gamma^{2(2 \beta-1)}\left[\left(\gamma-\frac{1}{\gamma}\right)^{2}+4 \rho^{2} \mu_{1}^{2 \alpha-1}\right] \\
& =\gamma^{4(\beta-1)}\left[\left(\gamma^{2}-1\right)^{2}+4 \rho^{2} \mu_{1}^{2 \alpha-1} \gamma^{2}\right]
\end{aligned}
$$

(a) Consider first the case where $2 \rho^{2} \mu_{1}^{2 \alpha-1} \geq 1$. We then write

(B.4) $\left(\gamma^{2}-1\right)^{2}+4 \rho^{2} \mu_{1}^{2 \alpha-1} \gamma^{2}=\gamma^{4}+2\left(2 \rho^{2} \mu_{1}^{2 \alpha-1}-1\right) \gamma^{2}+1 \geq \gamma^{4}+1$

so that for $\beta=1$ we obtain

$$
\gamma^{4(\beta-1)}\left[\left(\gamma^{2}-1\right)^{2}+4 \rho^{2} \mu_{1}^{2 \alpha-1} \gamma^{2}\right] \geq \gamma^{4}+1 \geq 1
$$


uniformly in $\gamma$, while for $\beta=0$ we obtain

$$
\gamma^{4(\beta-1)}\left[\left(\gamma^{2}-1\right)^{2}+4 \rho^{2} \mu_{1}^{2 \alpha-1} \gamma^{2}\right] \geq \frac{\gamma^{4}+1}{\gamma^{4}}=1+\frac{1}{\gamma^{4}} \geq 1
$$

uniformly in $\gamma$. Then (B.3), (B.5) for $\beta=1$ and (B.3), (B.6) for $\beta=0$ prove Lemma B.1, as desired, at least in case (a).

(b) Let now $2 \rho^{2} \mu_{1}^{2 \alpha-1}<1$. By completing the square we write

$$
\begin{aligned}
& \left(\gamma^{2}-1\right)^{2}+4 \rho^{2} \mu_{1}^{2 \alpha-1} \gamma^{2} \\
& \quad=\left(\gamma^{2}-1\right)^{2}+4 \rho^{2} \mu_{1}^{2 \alpha-1}\left(\gamma^{2}-1\right)+4 \rho^{2} \mu_{1}^{2 \alpha-1} \\
& \quad=\left[\left(\gamma^{2}-1\right)+2 \rho^{2} \mu_{1}^{2 \alpha-1}\right]^{2}+4 \rho^{2} \mu_{1}^{2 \alpha-1}\left(1-\rho^{2} \mu_{1}^{2 \alpha-1}\right)
\end{aligned}
$$

so that for $\beta=1$ dropping the square term we obtain

(B.8) $\gamma^{4(\beta-1)}\left[\left(\gamma^{2}-1\right)^{2}+4 \rho^{2} \mu_{1}^{2 \alpha-1} \gamma^{2}\right] \geq 4 \rho^{2} \mu_{1}^{2 \alpha-1}\left(1-\rho^{2} \mu_{1}^{2 \alpha-1}\right)>0$

uniformly in $\gamma$. Then (B.3), (B.8) prove Lemma B.1, as desired, for $\beta=1$ in the present case (b). Let now $\beta=0$. Then, similarly,

$$
\begin{aligned}
\gamma^{4(\beta-1)} & {\left[\left(\gamma^{2}-1\right)^{2}+4 \rho^{2} \mu_{1}^{2 \alpha-1} \gamma^{2}\right] } \\
& =1+\frac{1}{\gamma^{4}}+\frac{1}{\gamma^{2}} 2\left(2 \rho^{2} \mu_{1}^{2 \alpha-1}-1\right) \\
& =\left[\frac{1}{\gamma^{2}}+\left(2 \rho^{2} \mu_{1}^{2 \alpha-1}-1\right)\right]^{2}+1-\left(2 \rho^{2} \mu_{1}^{2 \alpha-1}-1\right)^{2} \\
& \geq 1-\left(2 \rho^{2} \mu_{1}^{2 \alpha-1}-1\right)^{2}=\left(2-2 \rho^{2} \mu_{1}^{2 \alpha-1}\right) 2 \rho^{2} \mu_{1}^{2 \alpha-1} \\
& =4 \rho^{2} \mu_{1}^{2 \alpha-1}\left(1-\rho^{2} \mu_{1}^{2 \alpha-1}\right)>0
\end{aligned}
$$

uniformly in $\gamma$. Then (B.3), (B.9) show Lemma B.1 also for $\beta=0$ in the present case (b). The proof of Lemma B. 1 is complete.

Note added in proof (August 1988) by R. T. The following paper has just appeared: On the mathematical model for linear elastic systems with analytic damping by F. Huang, SIAM J. Control \& Optimization, vol. 26, No. 3, May 1988. This paper likewise studies the problem of analytic generation of (1.1) when " $B$ is related in various ways to $A^{\alpha}$, $\frac{1}{2} \leq \alpha \leq 1$." This paper refers also to Huang's prior work in two papers in Acta Mathem. Sci. (apparently, in Chinese) appeared in 1985 and 1986, which refer to the case $\alpha=\frac{1}{2}$. These papers are not available as yet to the present authors. While Huang's work presents some overlap of results particularly with our prior paper [C-T.1] in the case $\alpha=\frac{1}{2}$, it does not contain however a result of the strength of our main Theorem 1.1 , let alone in the form presented in our Remark 1.3, for $\frac{1}{2}<\alpha \leq 1$. 
Our Theorem 1.1 is of global character (the constants $0<\rho_{1}, \rho_{2}<\infty$ are arbitrary), while Theorem 4.1 in Huang's paper is still of local character. In particular, Huang's Corollary 4.3 is contained in our Theorem 1.1.

\section{REFERENCES}

[B.1] A. V. Balakrishnan, Control of Flexible Flight Structures, paper dedicated to J. L. Lions on his sixtieth birthday, to be published by Gauthier-Villars, Paris, 1987.

[C-R.1] G. Chen and D. L. Russell, A Mathematical Model for Linear Elastic Systems with Structural Damping, Quart. Appl. Math., Jan. (1982), 433-454.

[C-T.1] S. Chen and R. Triggiani, Proof of two conjectures of G. Chen and D. $L$. Russell on Structural Damping for Elastic Systems, Proceedings of "Seminar in Approximation and Optimization" held at University of Havana, Cuba, January 12-14, 1987; Springer-Verlag Lecture Notes in Mathematics, 1988. Also, Proceedings of "First Conference in Communication and Control Theory" honoring A. V. Balakrishnan on his sixtieth birthday, held in Washington D. C. June 17-19, 1987, Optimization Software 1988.

[D-P.1] M. C. Delfour and M. P. Polis, On issues related to stabilization of large flexible structures, to appear in the series SIAM Frontiers in Applied Mathematics.

[D.1] N. Dunford, A survey of the theory of spectral operators, Bull. Amer. Math. Soc., 64, 5, (1958), 217-274.

[D-S.1] N. Dunford and J. Schwartz, Linear Operators, I (1958), II (1963), III (1971), Interscience Pubs, John Wiley, New York.

[F.1] H. O. Fattorini, The Cauchy Problem, Encyclopedia of Mathematics and its Applications, Addison-Wesley, Benjamin/Dummings Inc., Reading, Massachusetts

$01867,1983$.

[K.1] T. Kato, Perturbation of Linear Operators, Springer-Verlag, New York, 1966.

[K.2] S. G. Krein, Linear differential equations in Banach space, Trans. Amer. Math. Soc., Vol. 29, American Mathematical Society, Providence, RI 02904, 1971.

[L-M.1] J. L. Lions and E. Magenes, Nonhomogeneous Boundary Value Problems, Vol. I, Springer-Verlag, New York, 1972.

[L-T.1] I. Lasiecka and R. Triggiani, Finite rank, relatively bounded perturbations of $C_{0}$-semigroups, Part II: Spectrum allocation and Riesz basis in parabolic and hyperbolic feedback systems, Ann. Mat. Pura. Appl. IV, CXLIII (1986), 47-100.

[L-T.2] - Feedback semigroups and cosine operators for boundary feedback parabolic and hyperbolic equations, J. Differential Equations, 47 (1983), 246-272.

[P.1] A. Pazy, Semigroups of Operators and Applications to Partial Differential Equations, Springer-Verlag, New York, 1983.

[R.1] D. L. Russell, Mathematical Models for the Elastic Beam and Their ControlTheoretic Implications in Semigroups, Theory and Applications, Vol. II, H. Brezis, M. G. Crandall and F. Kappel, Editors, Pitman Research Notes in Mathematics Series, \#152. 
[R.2] On mathematical models for the elastic beam with frequency-proportional damping, preprint 1987.

[S.1] M. Schechter, Principles of Functional Analysis, Academic Press, New York 1971. sp

[T.1] R. Triggiani, On the Stabilizability Problem in Banach Space, J. Math. Anal. Appl., 52 (1975), 383-403. Addendum, J. Math. Anal. Appl., 56 (1976), 492-493.

[T.2] _ Improving Stability Properties of Hyperbolic Damped Equations by Boundary Feedback, Springer-Verlag Lecture Notes LNCIS (1985), 400-409.

[X.1] D. Xia, Spectral Theory of Hyponormal Operators, Birkhauser Verlag, BaselBoston-Stuttgart, 1983.

Received May 22, 1987 and in revised form February 3, 1988. Research partially supported by the Air Force Office of Scientific Research under Grant AFOSR 840365. The first author's research was performed in the U.S. under the Pao Yu-kong and Pao Zao-long Scholarship.

ZHEJIANG UNIVERSITY

HANGZHOU, ChINA

AND

UNIVERSITY OF VIRGINIA

Charlottesville, VA 22903 



\section{PACIFIC JOURNAL OF MATHEMATICS EDITORS}

\author{
V. S. VARADARAJAN \\ (Managing Editor) \\ University of California \\ Los Angeles, CA 90024 \\ HeRbert Clemens \\ University of Utah \\ Salt Lake City, UT 84112 \\ THOMAS ENRIGHT \\ University of California, San Diego \\ La Jolla, CA 92093
}

R. FINN

Stanford University

Stanford, CA 94305

HERMANN FLASCHKA

University of Arizona

Tucson, AZ 85721

VAUGHAN F. R. JONES

University of California

Berkeley, CA 94720

STEVEN KERCKHOFF

Stanford University

Stanford, CA 94305

\section{ROBION KIRBY}

University of California

Berkeley, CA 94720

C. C. MOORE

University of California

Berkeley, CA 94720

HAROLD STARK

University of California, San Diego

La Jolla, CA 92093

\section{ASSOCIATE EDITORS}
R. ARenS
E. F. BECKENBACH
B. H. NeumanN
F. WOLF
K. YOSHIDA (1906-1982)

\section{SUPPORTING INSTITUTIONS}
UNIVERSITY OF ARIZONA
UNIVERSITY OF OREGON
UNIVERSITY OF BRITISH COLUMBIA
UNIVERSITY OF SOUTHERN CALIFORNIA
CALIFORNIA INSTITUTE OF TECHNOLOGY
STANFORD UNIVERSITY
UNIVERSITY OF CALIFORNIA
MONTANA STATE UNIVERSITY
UNIVERSITY OF HAWAII
UNIVERSITY OF NEVADA, RENO
UNIVERSITY OF TOKYO
NEW MEXICO STATE UNIVERSITY
UNIVERSITY OF UTAH
OREGON STATE UNIVERSITY
WASHINGTON STATE UNIVERSITY
UNIVERSITY OF WASHINGTON 


\section{Pacific Journal of Mathematics}

\section{Vol. 136, No. $1 \quad$ November, 1989}

Robert Archbold and Frederic W. Shultz, Characterization of $C^{*}$-algebras with continuous trace by properties of their pure states $\ldots \ldots \ldots \ldots \ldots \ldots 1$

Shu Ping Chen and Roberto Triggiani, Proof of extensions of two conjectures on structural damping for elastic systems $\ldots \ldots \ldots \ldots \ldots \ldots$

Philip Throop Church and James Timourian, A nonlinear elliptic

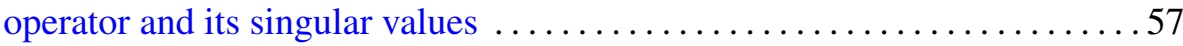

A. Gervasio Colares and Katsuei Kenmotsu, Isometric deformation of surfaces in $R^{3}$ preserving the mean curvature function $\ldots \ldots \ldots \ldots \ldots 71$

Fei Xu, A remark on spinor norms of local integral rotations. I . . . . . . . 81

Pedro Martinez Gadea and Ángel María Montesinos-Amilibia, Spaces of constant para-holomorphic sectional curvature $\ldots \ldots \ldots \ldots \ldots \ldots \ldots 5$

Guangxin Zeng, Homogeneous Stellensätze in semialgebraic geometry . . . . 103

Thomas Eric Hall, The isomorphism problem for orthodox semigroups . . . . 123

Mike Hoffman, Noncoincidence index, free group actions, and the fixed

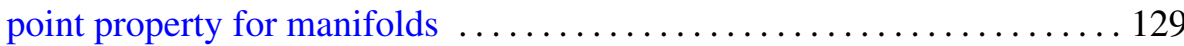

Terry Atherton Loring, The noncommutative topology of one-dimensional

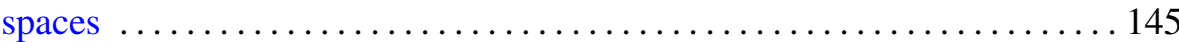

Haskell Paul Rosenthal and Alan Evan Wessel, The Krě̆ n-Mil'man property and a martingale coordinatization of certain nondentable convex sets

Yoshimi Saito, A remark on the limiting absorption principle for the reduced wave equation with two unbounded media 